\title{
Fra-1/AP-1 induces EMT in mammary epithelial cells by modulating Zeb1/2 and TGF $\beta$ expression
}

\author{
L Bakiri ${ }^{1,5}$, S Macho-Maschler ${ }^{2,5}$, I Custic ${ }^{3,6}$, J Niemiec ${ }^{2}$, A Guío-Carrión ${ }^{1}$, SC Hasenfuss ${ }^{1,7}$, A Eger ${ }^{4}$, M Müller ${ }^{2}$, H Beug ${ }^{2,3,8}$ \\ and EF Wagner*,1
}

Epithelial-to-mesenchymal transition (EMT) is essential for embryonic morphogenesis and wound healing and critical for tumour cell invasion and dissemination. The AP-1 transcription factor Fra-1 has been implicated in tumorigenesis and in tumour-associated EMT in human breast cancer. We observed a significant inverse correlation between Fra-1 mRNA expression and distant-metastasis-free survival in a large cohort of breast cancer patients derived from multiple array data sets. This unique correlation among Fos genes prompted us to assess the evolutionary conservation between Fra- 1 functions in EMT of human and mouse cells. Ectopic expression of Fra-1 in fully polarized, non-tumourigenic, mouse mammary epithelial EpH4 cells induced a mesenchymal phenotype, characterized by a loss of epithelial and gain of mesenchymal markers. Proliferation, motility and invasiveness were also increased in the resulting EpFra1 cells, and the cells were tumourigenic and efficiently colonized the lung upon transplantation. Molecular analyses revealed increased expression of $\operatorname{Tg} f \beta 1$ and the EMT-inducing transcription factors Zeb1, Zeb2 and Slug. Mechanistically, Fra-1 binds to the tgfb1 and zeb2 promoters and to an evolutionarily conserved region in the first intron of zeb1. Furthermore, increased activity of a zeb2 promoter reporter was detected in EpFra1 cells and shown to depend on AP-1-binding sites. Inhibiting TGF $\beta$ signalling in EpFra1 cells moderately increased the expression of epithelial markers, whereas silencing of zeb1 or zeb2 restored the epithelial phenotype and decreased migration in vitro and tumorigenesis in vivo. Thus Fra-1 induces changes in the expression of genes encoding EMT-related transcription factors leading to the acquisition of mesenchymal, invasive and tumorigenic capacities by epithelial cells. This study defines a novel function of Fra-1/AP-1 in modulating tgfb1, zeb1 and zeb2 expression through direct binding to genomic regulatory regions, which establishes a basis for future in vivo genetic manipulations and preclinical studies using mouse models.

Cell Death and Differentiation (2015) 22, 336-350; doi:10.1038/cdd.2014.157; published online 10 October 2014

Epithelial-to-mesenchymal transition (EMT) is a complex biological programme that occurs in physiological processes during embryonic development and wound healing as well as in pathological conditions, such as organ fibrosis and carcinogenesis. During EMT, cells lose epithelial features and acquire mesenchymal characteristics. The acquisition of a mesenchymal state by malignant cancer cells is associated with decreased cell-cell adhesion, and increased migratory and invasive properties, which are crucial for metastasis. ${ }^{1-5}$

The adherens junction (AJ) protein E-cadherin, encoded by $c d h 1$, is a central determinant of the epithelial state and its downregulation is the hallmark of EMT. A number of molecular pathways converging on E-cadherin have been implicated in EMT. Transcription factors (TF) of the Snail, Zeb and Twist families, initially identified as regulators of epithelial- mesenchymal plasticity during morphogenesis were shown to orchestrate EMT by controlling the expression of epithelial polarity determinants. ${ }^{4}$ These epithelial-to-mesenchymal transition transcription factors (EMT-TFs) are recruited to and repress the cdh1 1 promoter, ${ }^{6}$ often in the context of complex epigenetic modulations. ${ }^{7}$ A number of factors in the tumour environment, such as transforming growth factor beta (TGF $\beta$ ), growth factors and cytokines modulate EMT by regulating EMT-TFs. ${ }^{8}$ Dynamic regulatory circuits integrating EMT-TFs with numerous microRNAs ${ }^{9}$ additionally control epithelial plasticity (reviewed in Lamouille ${ }^{3}$ ).

Fos-related antigen 1 (Fra-1) is encoded by the FOS-like antigen 1 (Fos/1) gene. It is a member of the Fos family of basic leucine Zipper domain proteins that dimerize with Jun proteins to form the activator protein 1 (AP-1) TF. Several studies have

${ }^{1}$ Genes, Development and Disease Group, F-BBVA Cancer Cell Biology Programme, National Cancer Research Centre (CNIO), Madrid, Spain; ${ }^{2}$ Institute for Animal Breeding and Genetics, University of Veterinary Medicine, Vienna, Austria; ${ }^{3}$ Research Institute of Molecular Pathology, Vienna, Austria and ${ }^{4} \mathrm{IMC}$ University of Applied Sciences, Krems, Austria

${ }^{*}$ Corresponding author: EF Wagner, Genes, Development and Disease Group, F-BBVA-CNIO Cancer Cell Biology Programme, Spanish National Cancer Research Centre, Melchor Fernandez Almagro 3, Madrid 28029, Spain. Tel: +34 917328 000; Fax: +34 912 246 980; E-mail: ewagner@cnio.es

${ }^{5}$ These authors contributed equally to this work.

${ }^{6}$ Current address: European Molecular Biology Laboratory, Hamburg Unit, Hamburg, Germany.

${ }^{7}$ Current address: Dana-Farber Cancer Institute and Department of Cell Biology, Harvard Medical School, Boston, MA, USA.

8In memoriam (1945-2011).

Abbreviations: Fra-1, Fos-related antigen 1; Fosl1, FOS-like antigen 1; AP-1, activator protein 1; ERK, extracellular signal-regulated kinase; EMT, epithelial-tomesenchymal transition; EMT-TF, epithelial-to-mesenchymal transition transcription factor; AJ, adherens junction; TGF $\beta$, transforming growth factor beta; Zeb1, Zinc finger E-box-binding homeobox 1; GSEA, Geneset Enrichment Analysis; ChIP, chromatin immunoprecipitation; IHC, immunohistochemistry; qRT-PCR, quantitative reverse transcription PCR; GEMM, genetically engineered mouse model

Received 24.3.14; revised 31.7.14; accepted 29.8.14; Edited by G Melino; published online 10.10.14 
correlated Fra-1 expression with malignancy of human cancer. In breast cancer, levels of Fra-1 correlate with the mesenchymal features of aggressive variants, in both clinical specimens and cell lines, while Fra-1 expression in tumourassociated macrophages has been suggested to impact on mammary tumour progression. ${ }^{10,11}$ Strikingly, suppression of Fra-1 restores epithelial characteristics, including E-cadherin expression, and abrogates the invasive potential of human breast cancer cell lines. ${ }^{12,13}$ Furthermore, Fra-1 has been implicated in human breast cancer EMT through increased expression of the Snai2/Slug, Zinc finger E-box-binding homeobox 1 (Zeb1) and Zeb2 EMT-TFs, ${ }^{13-17}$ although the mechanisms how Fra-1 modulates EMT-TFs expression are not fully understood.

To further decipher how Fra-1 contributes to EMT and assess the evolutionary conservation of EMT-TF regulation by Fra-1, we used the non-tumorigenic murine mammary epithelial cell line, EpH4. ${ }^{18}$ The phenotypic plasticity of this cell line upon Ras/MAPK pathway manipulation has been extensively documented. ${ }^{19-22}$ Expression of $\mathrm{c}-\mathrm{Fos}^{18}$ or c$\mathrm{Jun}^{23}$ in $\mathrm{EpH} 4$ cells increases Fra- 1 mRNA and promotes EMT. Here we show that ectopic expression of Fra-1 is sufficient to trigger a mesenchymal, invasive and tumorigenic programme in EpH4 cells. Mechanistically, we uncover that Fra-1 induces critical changes in the expression of $\operatorname{tg} f b 1$ and EMT-TFs through direct binding to the tgfb1, zeb1 and zeb2 genomic regulatory regions.

\section{Results}

Inverse correlation between FOSL1 expression and metastasis-free survival in human breast cancer patients. Computational analysis using the $\mathrm{KM}$ plotter integrative bioinformatic interface ${ }^{24}$ revealed a significant correlation between high FOSL1 mRNA expression and poor metastasis-free survival (Figure 1a). This correlation extended to overall survival (Figure 1b) and was unique among Fos genes as the inverse trend was obtained when stratifying this very large cohort (1609 and 1105 patients, respectively) according to the expression of FOS (Figures 1c and d), FOSL2 or FOSB (Supplementary Figure S1). These findings are consistent with the recently documented c-Fos/ Fra-1 antagonism in human breast cancer stem cells ${ }^{25}$ and suggest a unique and therapeutically relevant function of Fra-1 in human breast cancer metastasis.

Fra-1 expression in EpH4 cells leads to epithelial-tofibroblastoid conversion, increased cell motility and invasiveness. To establish a basis for preclinical studies in genetically engineered mouse models (GEMMs), we investigated the evolutionary conservation between Fra-1 functions in human and mouse cell EMT. EpH4 and Ha-Rastransformed, EpRas murine mammary epithelial cells form polarized epithelial clusters in vitro. ${ }^{22}$ TGF $\beta$ induces cell cycle arrest and apoptosis in EpH4 cells and EMT in EpRas cells. ${ }^{22}$ Of the AP-1-forming proteins, Fra-1 is increased in EpRas cells and in EpRasXT cells, which have undergone EMT in vivo (Supplementary Figures S2a and b). EpH4 cell lines constitutively expressing Fra-1 (EpFra1) were established, and two lines, expressing comparable Fra-1 levels as EpRas cells, were further analysed (Figure 2a). EpFra1 cells grew scattered and adopted fibroblastoid morphology. Numerous cell protrusions were observed that accumulated actin, reminiscent of motile mesenchymal cells (Figure $2 b$, Supplementary Figure S2c). Molecular analyses revealed a striking loss of epithelial markers. Most notably, E-cadherin protein and mRNA expression was decreased (Figures 2c and d, Supplementary Figure S2c). Consistent with the crucial role of E-cadherin in maintaining AJ integrity, AJ proteins such as $\alpha-, \beta-, \gamma$ - and p120-catenin were decreased, while their mRNA expression was little affected (Figures 2c and $\mathrm{d}$ ). The cytokeratins krt7 and krt8, the tight junction proteins $c r b 3$ and $c / d n 23$ and the integrins $\beta 2, \beta 5$ and $\beta 6$ were also downregulated (Supplementary Figures S2d and e). Conversely, EpFra1 cells expressed fibronectin and vimentin, two mesenchymal markers undetectable in $\mathrm{EpH} 4$ cells (Figures 2c and d, Supplementary Figure S2c). Other mesenchymal genes, such as cdh2, cdh3, s100a4, spp1, the $a 5$ and $\beta 1$ integrins, and several matrix metalloproteases were increased (Figure 2d, Supplementary Figures S2d and e). Immunohistochemistry (IHC) confirmed decreased AJ proteins and increased fibronectin (Supplementary Figure S2c). Higher cell counts were measured in EpFra1 cultures (Figure 2e), and EpFra1 cells displayed increased motility and invasiveness in Transwell and Matrigel assays (Figures $2 f$ and g). Importantly, knockdown of Fra-1 in EpFra1 cells resulted in the re-acquisition of epithelial morphology (Supplementary Figures S3a and b) and E-cadherin upregulation, while other epithelial and mesenchymal markers were restored to variable extent (Supplementary Figures S3c and e). This implies that constant ectopic Fra- 1 expression is necessary for maintaining E-cadherin repression. Decreased E-cadherin mRNA was also observed in mouse NMuMG mammary epithelial cell pools transduced with a Fra1ERexpressing retrovirus and treated with 4-hydroxyTamoxifen to activate the Fra1ER fusion protein (Supplementary Figure S3f). In conclusion, Fra-1 decreases E-cadherin expression and triggers morphological, molecular and functional features characteristic of EMT in EpH4 cells.

Expression profiling identifies EMT-related global changes associated with Fra-1. Gene expression profiling was performed to gain insight into the molecular changes triggered by Fra-1 expression. Approximately 400 genes were upregulated and 250 decreased by at least two fold in EpFra1 cells, when compared with $\mathrm{EpH} 4$ cells (Supplementary Figure S4a). We used the Molecular Signatures Database available on the Gene Set Enrichment Analysis (GSEA) web resource to analyse the genes affected by Fra-1 and compute overlaps with curated expression signatures, pathway databases and TF-binding sites. ${ }^{26}$ Remarkably, GSEA showed a significant overlap of the Fra-1-derived gene sets with published gene expression profiles associated with cellular transformation, mesenchymal breast cancer cells and invasive breast carcinomas (Table 1). The most significant pathways in the upregulated gene set were the AP-1, Interleukin-6 and Hif1a/hypoxia pathways, as well as genes involved in extracellular matrix organization and degradation (Table 1a). The Fra-1 

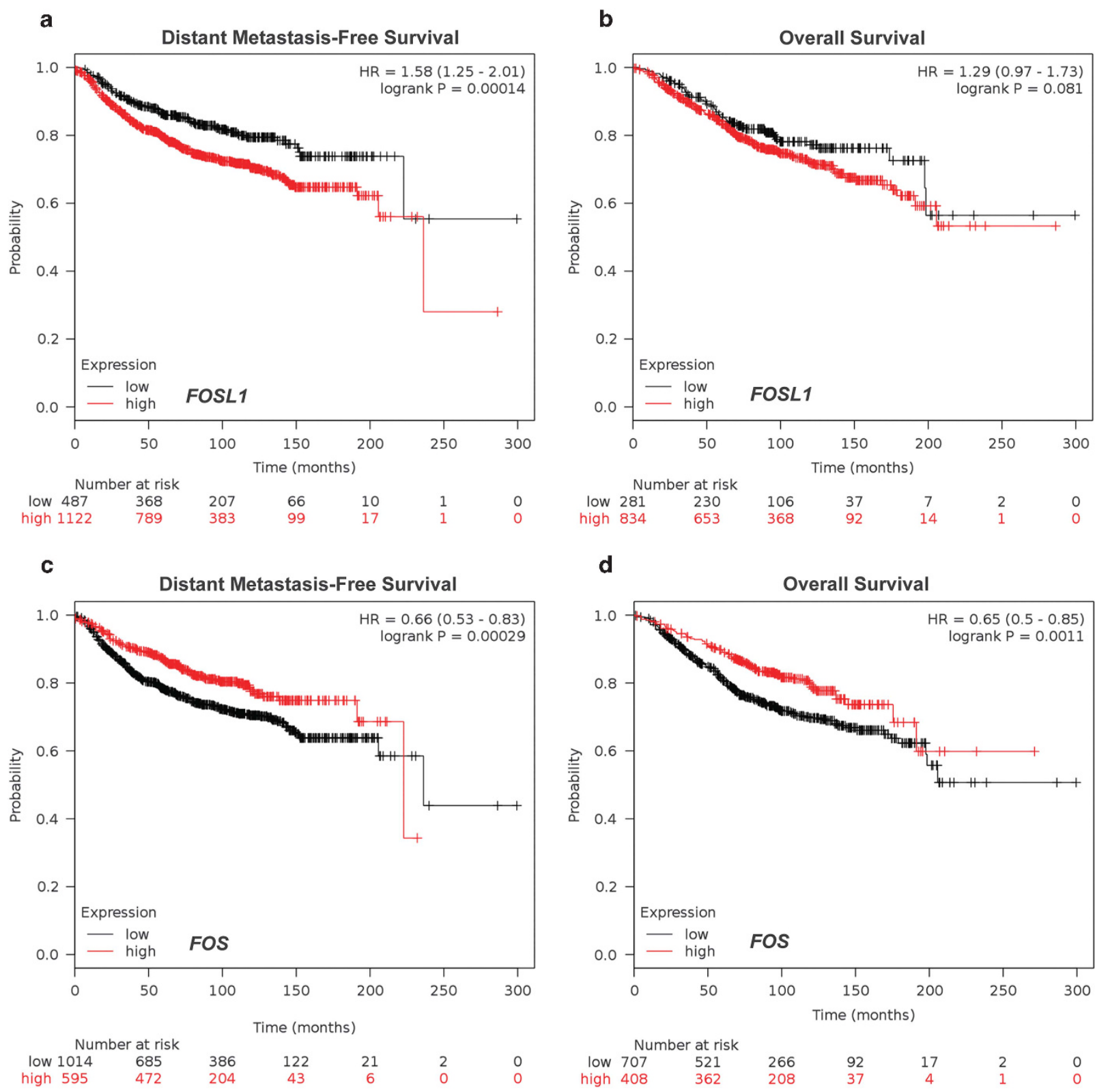

Figure 1 Prognostic value of FOSL1 and FOS expression in breast cancer patients. (a) Correlation of the levels of FOSL1 and FOS expression and prognosis in human breast cancer patients using the Kaplan-Meier (KM) plotter integrative data analysis tool:;4 $\mathrm{http}: / /$ www.kmplot.com. Shown are KM survival plots for patient samples classified as having high (red) or low (black) (a and b) FOSL1 or (c and d) FOS median expression to assess metastasis-free (DMFS: a, c) or overall (OS: b, d) survival. Hazard ratio (HR) with $95 \%$ confidence intervals and log-rank $P$-value are displayed, and the number of patients at risk in the high and low groups are indicated for each time point. The total number of patients with available clinical data is: DMFS, $n=1609$; and OS, $n=1105$

downregulated gene set was enriched for genes and pathways involved in cell junction organization and tight junction interactions (Table 1b), with a significant overlap with genes downregulated in human mammary epithelial cells undergoing EMT upon E-cadherin knockdown. ${ }^{27}$ Genes and pathways connected with apoptosis or p53 also appeared deregulated and some gene expression changes, such as dusp1, Fas, FasL and zmat3, were confirmed by quantitative reverse transcription PCR (qRT-PCR; Table 1b and Supplementary Figure S4b). Finally, a significant correlation was found with gene sets characteristic of mesenchymal and invasive breast cancer, as well as mammary stem cells, consistent with the recently emerging relationship between EMT and stemness in breast cancer. ${ }^{28}$ Collectively, these data support the notion that Fra-1 induces gene expression programmes in $\mathrm{EpH} 4$ cells characteristic of EMT, transformation and invasiveness.

Fra-1 expression in EpH4 cells induces tumorigenesis and metastasis. The consequences of Fra-1 expression were assessed in xenografts. In contrast to EpRas cells, EpH4 cells form small benign nodules at a low frequency, when implanted into mammary glands and are not able to colonize distant organs, even when delivered in the vascular system. ${ }^{22}$ 
a
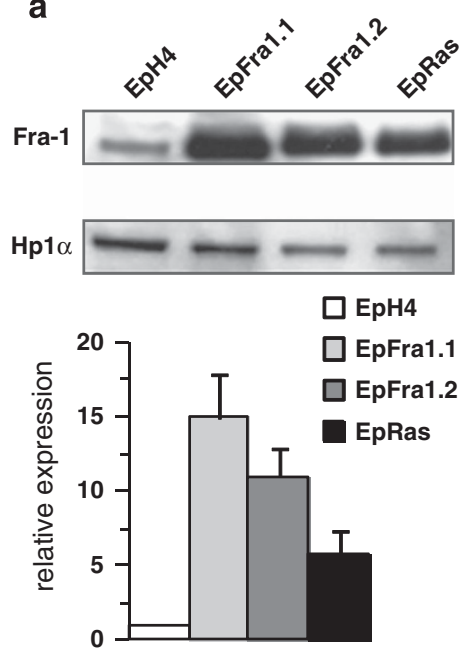

C

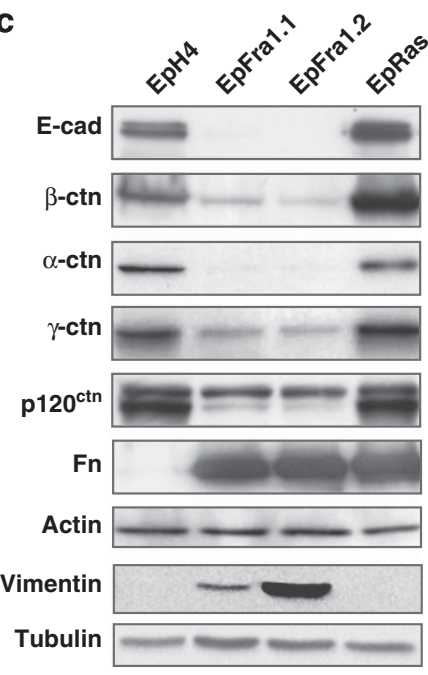

e

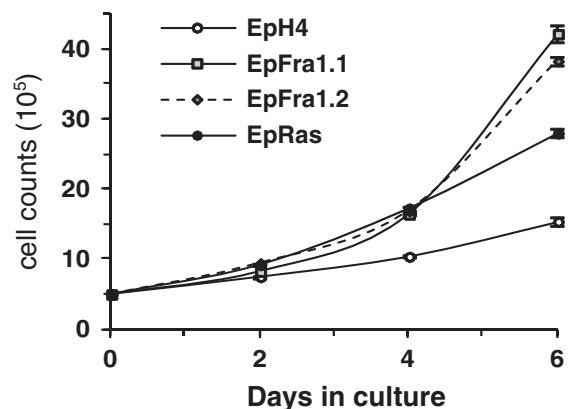

b
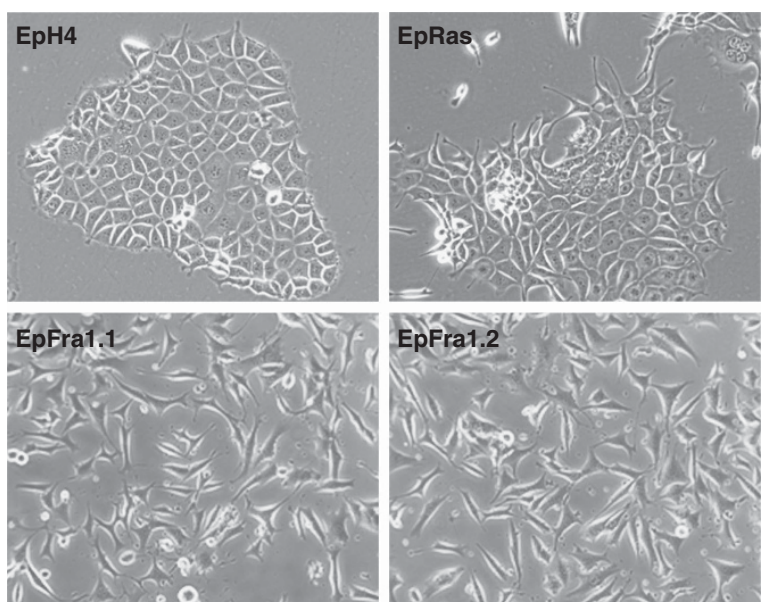

a
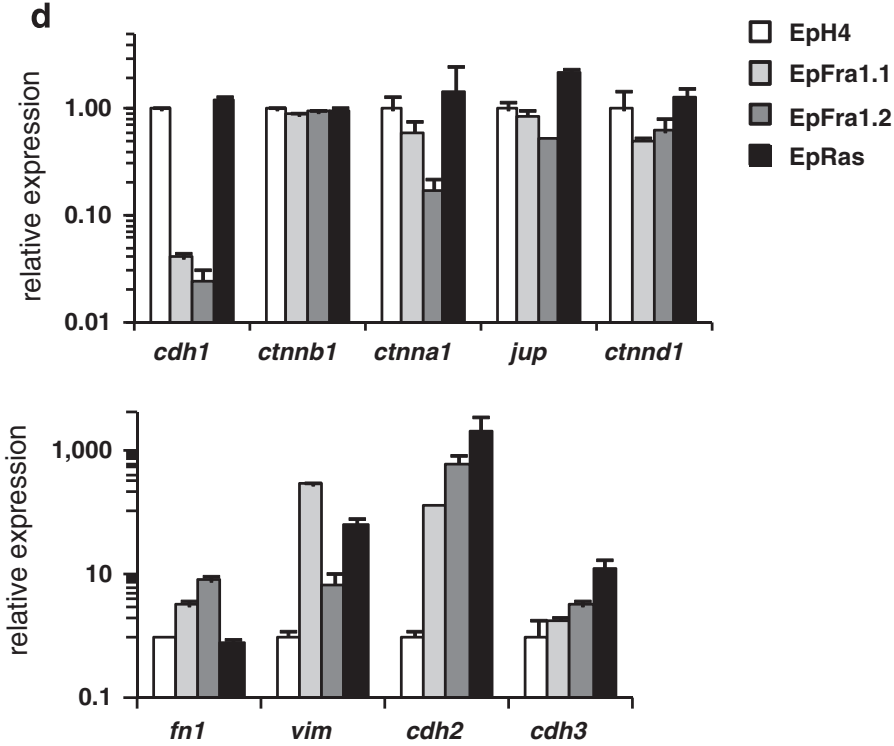

$\mathbf{f}$
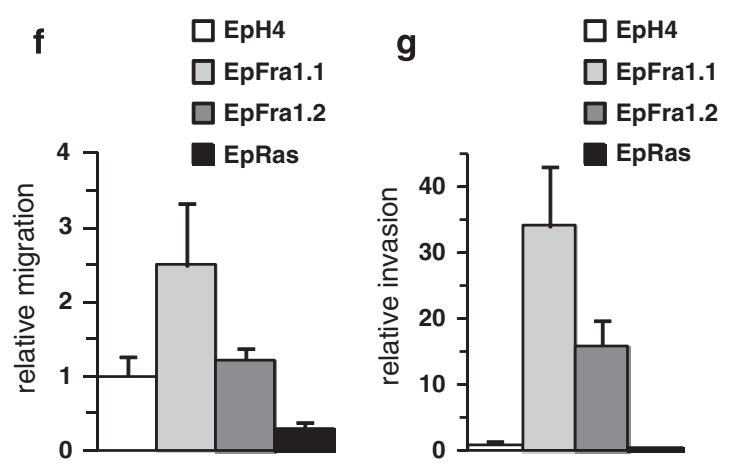

Figure 2 Fra-1 expression in EpH4 cells leads to fibroblastoid conversion and increased cell motility and invasiveness in vitro. (a) Analysis of Fra-1 protein (Top) and mRNA (Bottom) in EpH4-derived cells. Heterochromatin Protein 1a ( $\mathrm{Hp} 1 \alpha)$ is used to control loading. Expression in parental EpH4 is set to 1. (b) Cell morphology of EpH4-derived cells. (c) Western blotting and (d) qRT-PCR analysis of epithelial and mesenchymal markers in EpH4-derived cells. Actin and Tubulin are used to control loading in panel (c), and EpH4 are set to 1 in panel (d). Bars = mean \pm S.D., $n=2$. In vitro (e) proliferation, (f) migration and relative invasion (g) of EpH4-derived cells. Bars $=$ mean \pm S.D., $n=3$. cdh1, cdh2, cdh3, fn1, vim, ctnna1, ctnnb1, jup and ctnnd1 encode for E-cadherin, N-cadherin, P-cadherin, Fibronectin (Fn), Vimentin, $\alpha$-catenin $(\alpha$-ctn), $\beta$-catenin ( $\gamma$-ctn) and p120-catenin (p120ctn), respectively 
Table 1 Gene Set Enrichment Analysis (GSEA) of EpFra1 cells a. Upregulated set

No. of genes in comparison (n): 381

Collection

Description

$P$-value

CP: canonical pathways

Genes involved in interleukin-6 signalling

Genes involved in extracellular matrix organization

$5.58 e^{-5}$

$8.69 e^{-5}$

IL-6 signalling pathway

Osteopontin-mediated events

Toll-like receptor signalling pathway

HIF-1-alpha transcription factor network

AP-1 transcription factor network

IL-6-mediated signalling events

ATF-2 transcription factor network

Validated transcriptional targets of AP1 family members Fra1 and Fra2

Genes involved in degradation of the extracellular matrix

CGP: chemical and genetic perturbations The 'adult tissue stem' module: genes coordinately upregulated in a compendium of adult tissue stem cells

Genes upregulated in invasive ductal carcinoma (IDC) relative to ductal carcinoma in situ (DCIS, non-invasive).

Genes upregulated during epithelial-to-mesenchymal transition (EMT) induced by

TGFB1 in the EpH4 cells transformed by HRAS)

Upregulated genes in the cancer gene signature, representing a gene signature of cellular transformation

Genes downregulated in MCF7 cells at $24 \mathrm{~h}$ of estradiol treatment

Genes upregulated in MCF7 cells under hypoxia conditions

Genes upregulated upon overexpression of PARVB in MDA-MB-231 cells cultured in 3D Matrigel only

Genes downregulated in luminal-like breast cancer cell lines compared with the mesenchymal-like ones

Genes upregulated in MCF7 cells treated with hypoxia mimetic DMOG

Genes downregulated in luminal-like breast cancer cell lines compared with the basal-like ones

Genes upregulated in response to both hypoxia and overexpression of an active form of HIF1A

Genes consistently upregulated in mammary stem cells both in mouse and human $\quad 9.59 e^{-12}$ species

\section{b. Downregulated set}

No. of genes in comparison (n): 246

Collection

Description

$P$-value

$\mathrm{CP}$ : canonical pathways

Genes involved in cell-cell junction organization

Genes involved in tight junction interactions

Genes involved in cell junction organization

Genes involved in apoptotic cleavage of cell adhesion proteins

Genes involved in cell-cell communication

CGP: chemical and genetic perturbations Genes consistently downregulated in mammary stem cells both in mouse and human species

Genes downregulated in HMLE cells after E-cadhedrin knockdown by RNAi

Genes upregulated in luminal-like breast cancer cell lines compared with

the mesenchymal-like ones

Genes upregulated in MDA-MB-231 cells after knockdown of ZEB1 by RNAi

Genes upregulated in basal-like breast cancer cell lines as compared with the mesenchymal-like ones

Myb-regulated genes in MCF7 and lung epithelial cell lines overexpressing MYBL2,

MYBL1 or MYB

Genes associated with a migration rate of 40 human bladder cancer cells

Genes with promoters occupied by SMAD2 or SMAD3 in HaCaT cells (keratinocyte) $7.43 \mathrm{e}^{-11}$ according to a ChIP-chip analysis

Comparative analysis of the gene sets upregulated (a) or downregulated (b) in EpFra1 cells compared with EpH4 cells with the Molecular Signatures Database. The most significant overlaps with curated expression signatures from the CGP (with focus on mammary-related signatures) and the CP collections are presented, relevant keywords highlighted in bold, and the $P$-values are indicated

EpFra1 cells produced large fast growing and highly vascularized tumours when injected orthotopically (Figure 3a). High proliferative index was apparent by Ki67 IHC (Figure 3b). Similar to EpRas, EpFra1 tumours were not metastatic but efficiently colonized the lungs when delivered intravenously, with EpFra1 cells forming roughly three times less tumours than EpRas cells (Figure 3c). Tumours from both cell types were histologically indistinguishable, and IHC confirmed Fra-1 and fibronectin expression and E-cadherin suppression in transplanted EpFra1 cells, while EpRasderived tumours lost E-cadherin expression as previously reported (Figure 3d). 
a

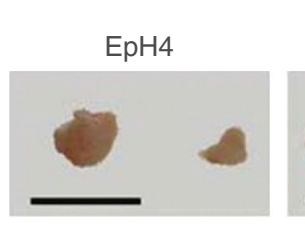

EpFra1.1

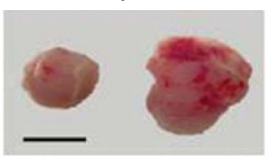

EpFra1.2

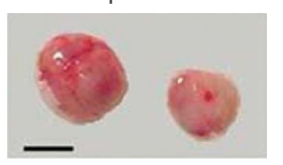

ํㅡㄴ

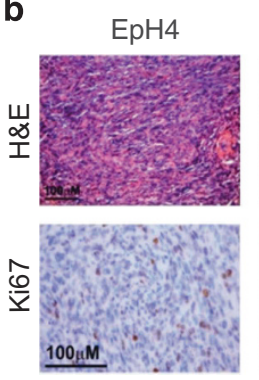

EpFra1.1
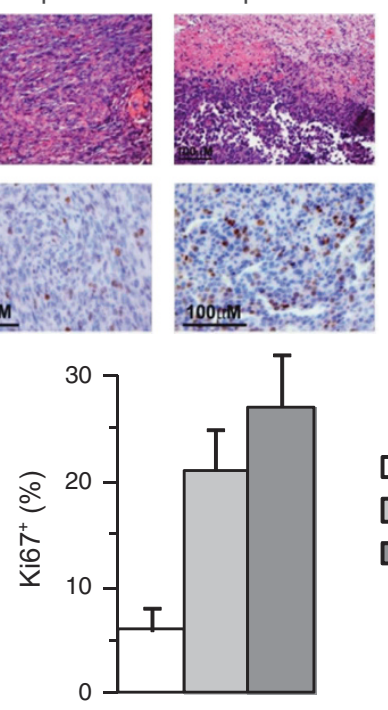

EpFra1.2

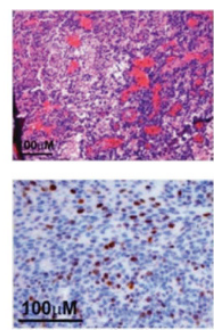

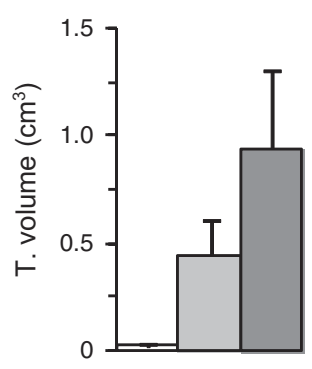

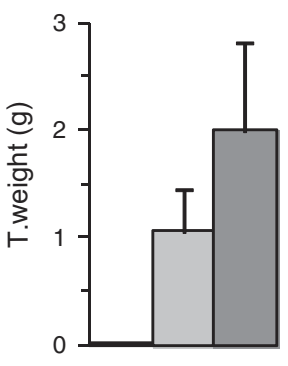

$\square \mathrm{EpH} 4$

$\square$ EpFra1.1

$\square$ EpFra1.2
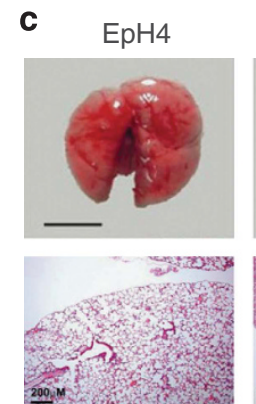

EpFra1.1
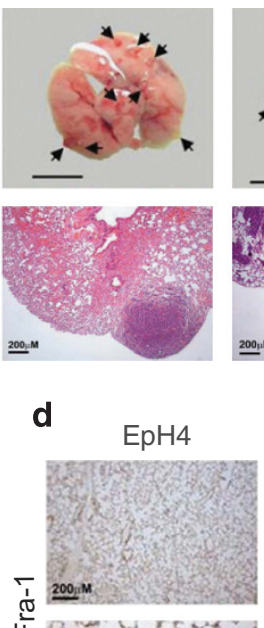

फे
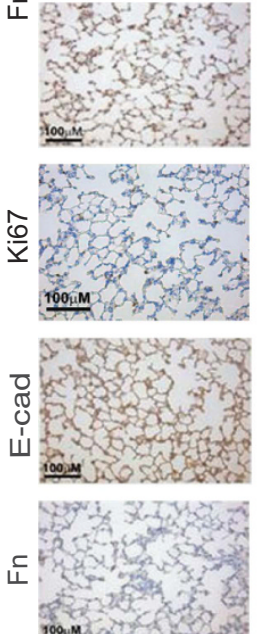

EpFra1.2
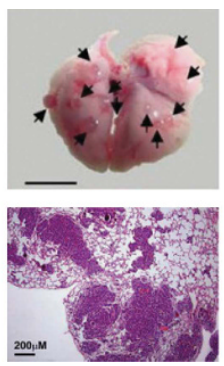

EpFra1.1
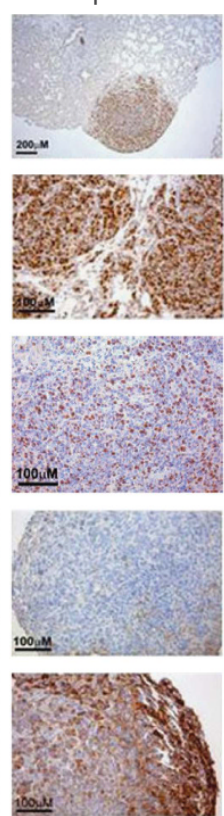

EpRas
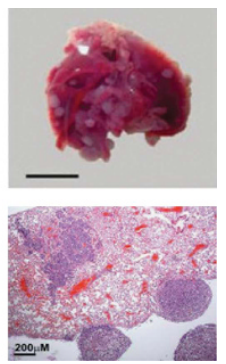

EpFra1.2
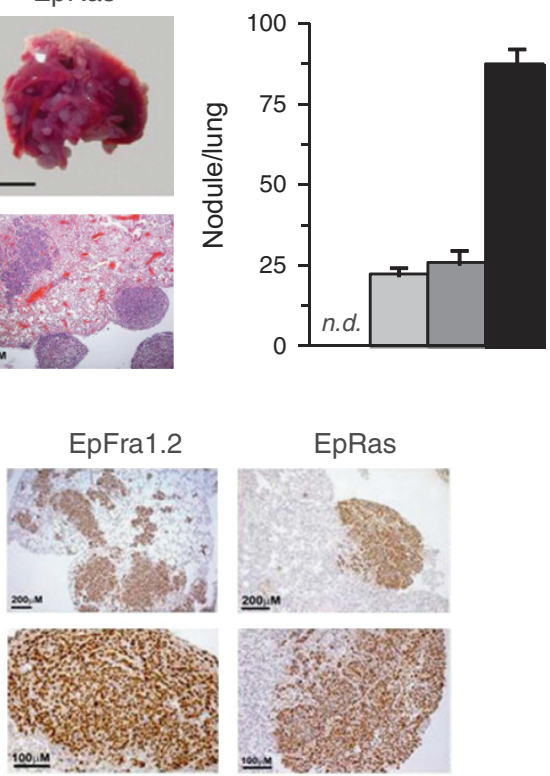

$\square \mathrm{EpH} 4$

$\square$ EpFra1.1

$\square$ EpFra1.2

EpRas

EpFra1.1

口 EpFra1.2

Figure 3 Fra-1 expression in $\mathrm{EpH} 4$ cells induces tumorigenesis and metastasis in xenograft models. (a) Top: Representative photographs of mammary gland tumours 14 days after injection of $\mathrm{EpH} 4$ or EpFra1 cells. Bar $=1 \mathrm{~cm}$. Bottom: Quantification of tumour volume and weight. Bars $=$ mean \pm S.D., $n=3$ mice/2-4 mammary glands per cell type. (b) H\&E staining and Ki67 IHC of tumour sections. Quantification of Ki67 is presented as the percentage of total nuclei. Bars $=$ mean \pm S.D., $n=3$. (c) Representative photographs of lungs $(\mathrm{Bar}=1 \mathrm{~cm})$ and $\mathrm{H \& E}$ staining of lung sections from mice injected with EpH4, EpFra1 or EpRas cells. Quantification of macroscopically visible nodules (indicated by arrows) is presented on the right. Bars $=$ mean \pm S.D., $n=4$. ND: no visible nodules. (d) Fra-1, Ki67, E-cadherin and Fibronectin (Fn) IHC on lung sections 
TGF $\beta$ pathway activation in Fra-1-expressing EpH4 cells. The lung colonization potential of EpRas cells strictly correlates with the ability to undergo TGF- $\beta$-induced EMT. ${ }^{19}$ GSEA revealed a significant overlap between the Fra-1derived gene sets and the genes upregulated in EpRas cells upon TGF- $\beta$-induced EMT ${ }^{21}$ (Table 1a). Genes associated with $\mathrm{c}-\mathrm{Myb}^{29}$ or with promoters occupied by Smad2 $/ 3,{ }^{30}$ two TFs connected to TGF- $\beta$ and EMT, ${ }^{31,32}$ were also enriched (Table 1b). Furthermore, tgfb1 mRNA was increased in EpFra1 cells and decreased upon Fra-1 knockdown, while tgfb2, tgfb3, tgfbr1 and tgfbr2 were little affected (Figure 4a, Supplementary Figures $\mathrm{S} 5 \mathrm{a}$ and b). Increased TGF $\beta$ production was also measured in EpFra1 cell conditioned medium (Figure 4b), and increased c-Myb was detected in EpFra1 nuclear extracts (Figure $4 \mathrm{c}$ and Supplementary Figure S5c). Increased TGF $\beta$-pathway activity in EpFra1 cells was further confirmed by Smad2/3 reporter assays (Figure 4d). Putative AP-1-binding sites were described in the murine tgfb1 promoter $^{33}$ (Figure 4e). Chromatin immunoprecipitation (ChIP) demonstrated that Fra-1 antibodies efficiently immunoprecipitated two chromatin fragments containing the putative AP- 1 sites (Figure $4 \mathrm{f}$ and Supplementary Figure S5d), thus indicating that increased tgfb1 expression is likely due to direct transcriptional regulation by Fra-1-containing AP-1 dimers. Inhibiting TGF $\beta$ signalling using a TGBFR1 inhibitor modestly increased the expression of epithelial markers (Figures $4 \mathrm{~g}$ and $\mathrm{h}$ ) but had little effect on mesenchymal genes or cell morphology (Figure 4h, Supplementary Figure S5e and data not shown). Thus, increased TGF $\beta$ signalling contributes but is likely not essential for EMT in Fra-1-expressing cells.

A Zeb1/2 molecular signature in EpFra1 cells. We next probed Fra-1 interaction with EMT-TFs. The two members of the Zeb family were found upregulated in the microarray analysis, by qRT-PCR and by western blotting (Supplementary Figure S6a, Figures $5 \mathrm{a}$ and b). Slug, encoded by snai2, was also increased (Figures $5 a$ and b), whereas mRNA of snai1/Snail was moderately elevated and the protein undetectable (Figure $5 \mathrm{a}$ and data not shown). Furthermore, constant Fra-1 expression was required for increased Zeb1, Zeb2 and Slug (Supplementary Figures S6b and c). GSEA revealed that the promoters of the downregulated genes were enriched with TRE/CRE motifs, bound by AP-1, but also with E-boxes bound by Zeb/Slug proteins (Supplementary Table S1). Importantly, a significant overlap was found between the EpFra1 downregulated gene set and the genes upregulated in MDA-MB-231 cells following ZEB1 knockdown, ${ }^{34}$ as well as genes modulated by ectopic ZEB2 expression in A431 cells ${ }^{35}$ (Table $1 \mathrm{~b}$, Supplementary Figure S6d). Besides E-cadherin, decreased expression of several Zeb1/Zeb2 transcriptional targets, including polycistrons encoding for the miR-200 family, was confirmed by qRTPCR (Figure $5 \mathrm{c}$ ) and the expression of Zeb targets was largely restored upon Fra-1 knockdown (Supplementary Figure S6e). Zeb1 and Zeb2 were not affected by TGBFR1 inhibitor treatment, unlike snai2, further supporting that Fra-1 is responsible for the increased Zeb1 and Zeb2 expression (Supplementary Figures S7a and b). Finally, increasing Fra-1 expression in EpRas cells by retroviral gene transfer led to decreased E-cadherin, increased vimentin and a striking mesenchymal morphology (Supplementary Figures S7c-e). Increased Zeb1 and Zeb2 expression and downstream signalling was confirmed by qRT-PCR and gene expression profiling (Supplementary Figures S7f and g). All together, these data imply that Zeb1 and Zeb2 are the most relevant EMT-TF for the EMT phenotype of EpH4-derived cells.

Zeb1 and Zeb2 are direct transcriptional targets of Fra-1. We investigated the molecular basis for increased Zeb1/2 in EpFra1 cells. Expression of miR-200 family members that target Zeb1/236 was also decreased in EpRas cells that do not display increased Zeb proteins (Figures $5 \mathrm{~b}$ and c). Similarly, while miR-221/222, modulated by Fra-1 and Slug in human breast cancer cells, ${ }^{15,37}$ increased in EpFra1 cells, the expression of the miR-221/222 target and Zeb2 repressor Trps1 was decreased in both EpFra1 and EpRas cells (Supplementary Figures S8a and b). Thus miR-200 and miR-221/222/Trps1 are not sufficient to explain increased zeb2 expression. A putative AP-1-binding site has been described in the human zeb1 promoter $^{38}$ and additional sites were found in the first intron, all largely conserved in the mouse sequence (Figure $5 \mathrm{~d}$ ). On the other hand, several putative AP-1 sites were found in the P3 promoter of zeb2, which directs Zeb2 expression in mouse mammary cells ${ }^{39}$ (Figure 5d). ChIP demonstrated that Fra-1 antibodies immunoprecipitated chromatin fragments containing the AP-1 sites of the intronic but not the zeb1 promoter (Figure 5e, Supplementary Figure S8c). Fragments containing the AP-1 sites of zeb2 promoter were also enriched (Figure $5 f$, Supplementary Figure S8d). Consistently, increased luciferase activity was measured in EpFra1 cells transfected with a wild-type zeb2 promoter luciferase reporter, which was abolished upon mutation of the three AP-1 sites (Figure 5g). Collectively, these data indicate that a direct transcriptional regulation by Fra-1-containing AP-1 dimers leads to increased zeb1 and zeb2 expression in EpFra1 cells.

Zeb1 and Zeb2 are essential for EMT in EpFra1 cells. Stable lines expressing shRNA targeting either zeb1 (EpFra1-sZ1) or zeb2 (EpFra1-sZ2) were next derived from EpFra1.2 cells. Zeb1 protein and mRNA expression was decreased in EpFra1-sZ1 but not in EpFra1-sZ2 cells (Figure 6a). On the other hand, Zeb2 protein and mRNA expression was decreased in EpFra1-sZ2 cells (Figure 6a, Supplementary Figure S9a). Strikingly however, EpFra1-sZ1 cells had decreased Zeb2 mRNA and protein (Figure 6a). Decreased Zeb2 mRNA was observed using five independent Zeb1 shRNAs (Supplementary Figure S9b). Conversely, EpH4-FosER cells expressing ZEB1 $1^{40}$ had increased Zeb2 mRNA in the absence of 4-hydroxyTamoxifen (Supplementary Figures S9c and d). This indicates that Zeb1 contributes to controlling Zeb2 expression in EpH4-derived cells. Importantly, while Fra-1 expression was unaffected (Figures 6a and c), EpFra1-sZ1 and EpFra1-sZ2 cells reacquired an epithelial morphology, which was more obvious in EpFra1-sZ1 cells (Figure 6b). E-cadherin and most epithelial markers, miR-200 and a subset of integrins, were restored to levels comparable to $\mathrm{EpH} 4$ cells, consistent with 
a

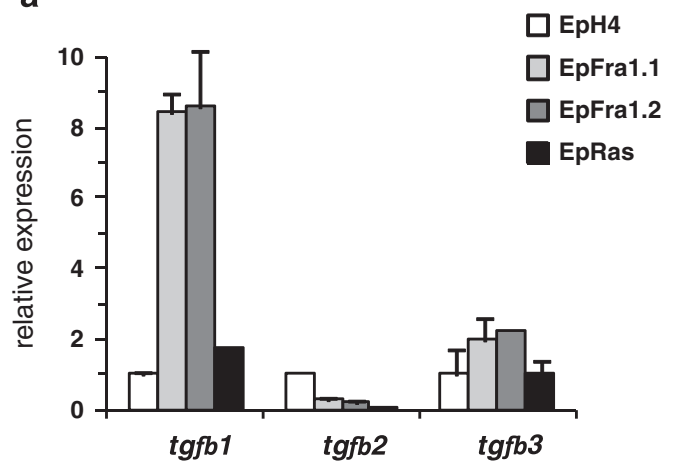

C

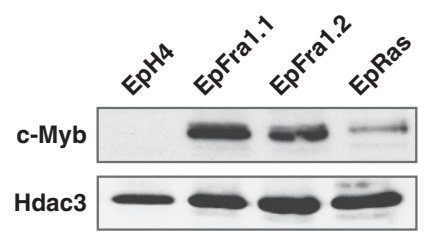

e
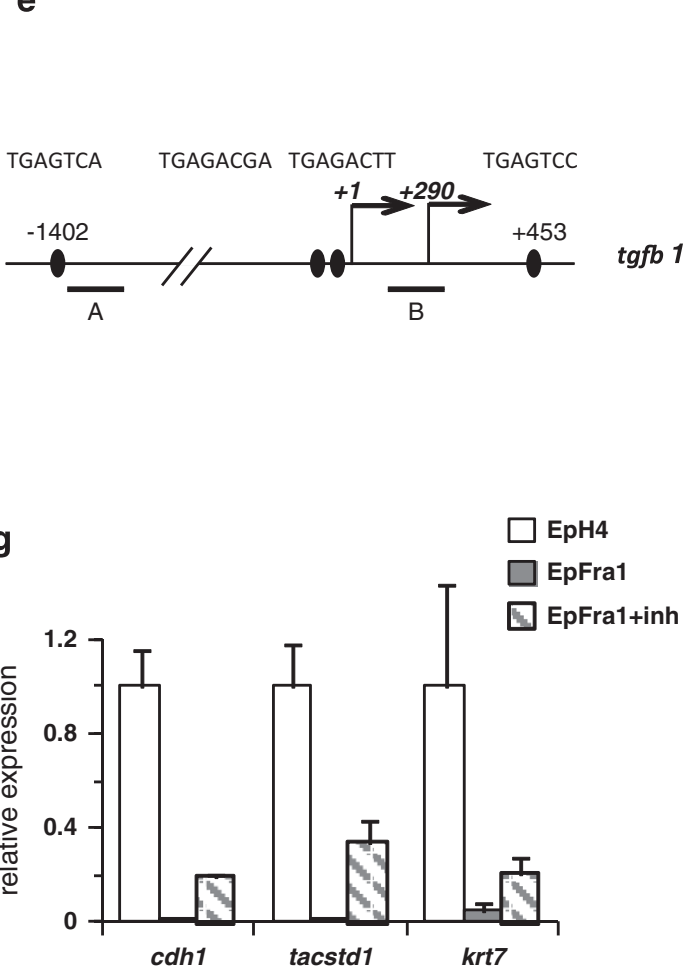

b

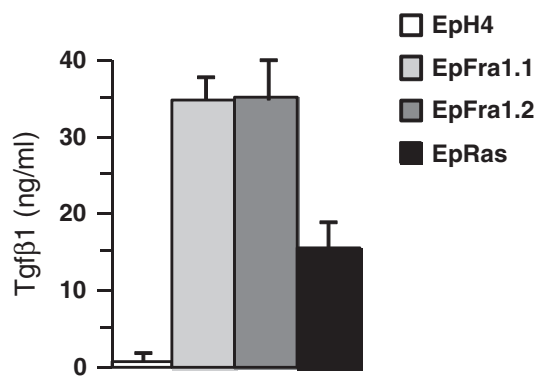

d

$\square$ EpH4

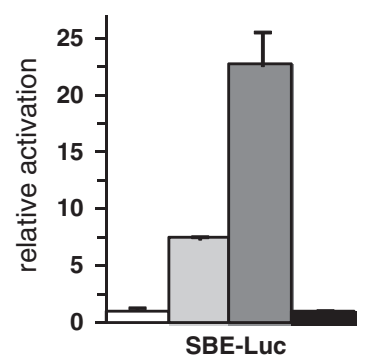

$\square$ EpFra1.1

$\square$ EpFra1.2

EpRas

f
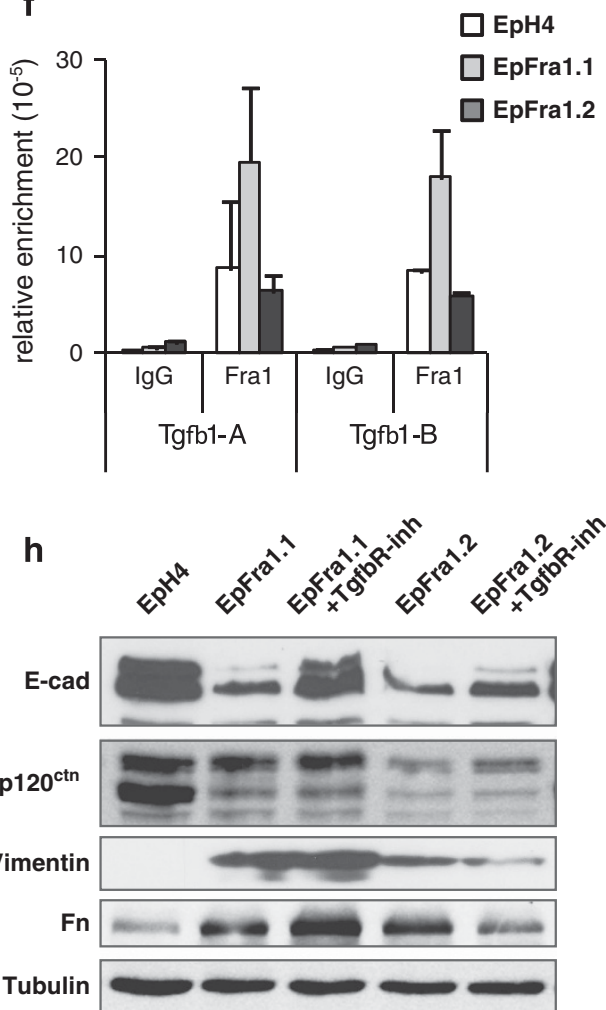

Figure 4 TGF $\beta$ pathway activation in Fra-1-expressing EpH4 cells - direct regulation of tgfb1 expression by Fra-1. (a) qRT-PCR analysis of tgfb gene expression in EpH4-derived cells. Bars $=$ mean \pm S.D., $n=2$, expression in EpH4 is set to 1. (b) ELISA quantification of TGF $\beta 1$ in the conditioned medium of EpH4-derived cells. Bars $=$ mean \pm Sd, $n=3$. (c) Western blotting analysis of c-Myb expression in nuclear extracts from EpH4-derived cells. Hdac3 is used to control loading. (d) Smad-reporter activity in EpH4-derived cells. Bars $=$ mean \pm S.D., $n=2$, relative light units in EpH4 is set to 1. (e) Scheme of the murine tgfb1 promoter. Transcription start sites and putative AP-1-binding elements are indicated. ChIP amplicons are depicted. (f) ChIP analysis of the tgfb1 promoter in EpH4-derived cells using $\alpha$-Fra-1 or lgG. Enrichment relative to input for each amplicon in one representative experiment is shown. Bars $=$ mean \pm S.D., $n=2$. Effects of TgfbR inhibitor treatment ( 5 days) on epithelial and mesenchymal markers in EpFra1 cells assessed by (g) qRT-PCR and (h) western blotting. Bars $=$ mean \pm S.D., $n=2$. Expression in $\mathrm{EpH} 4$ is set to 1, and the mean results of the two EpFra1 lines are averaged. Tubulin is used to control loading 

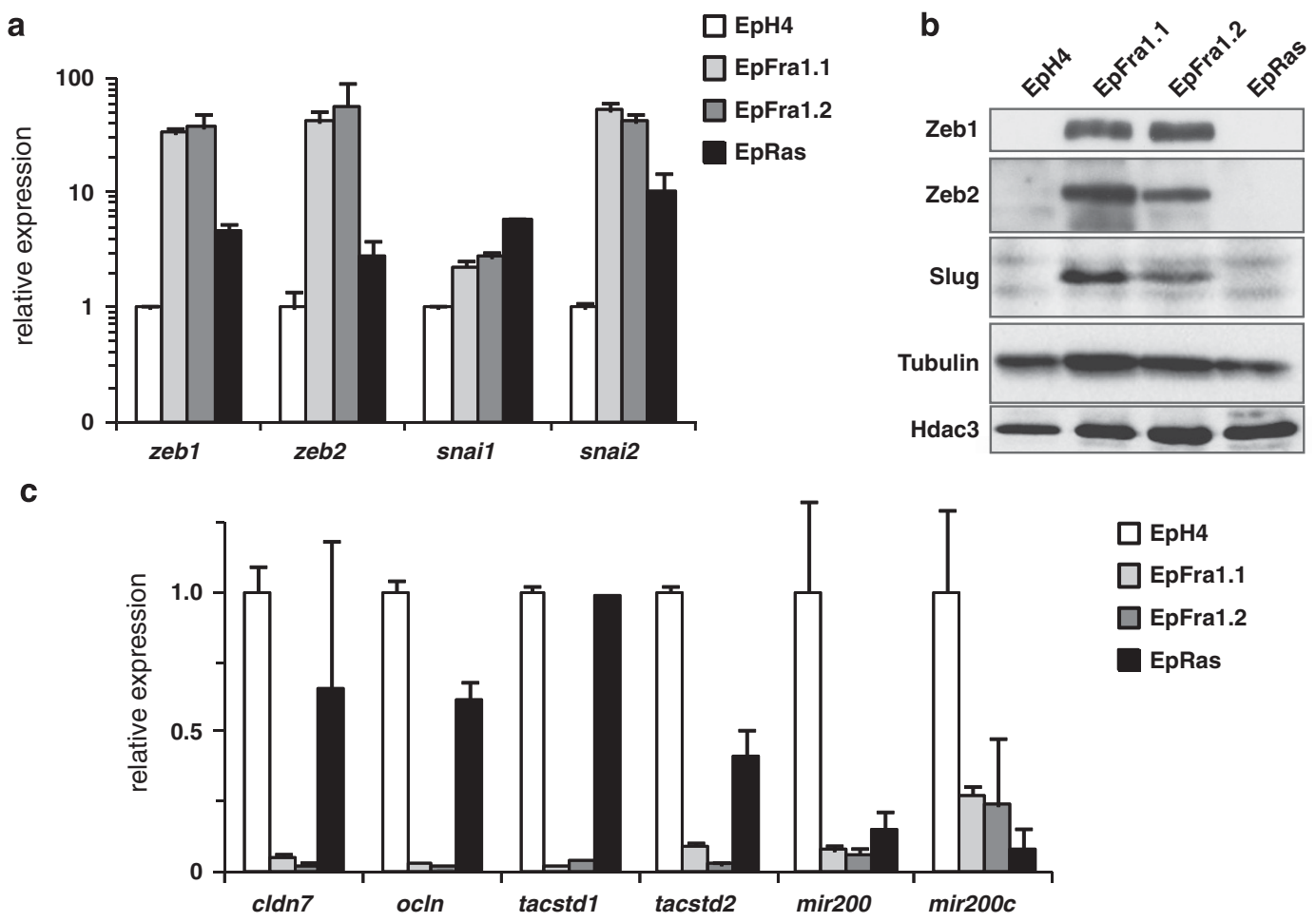

d
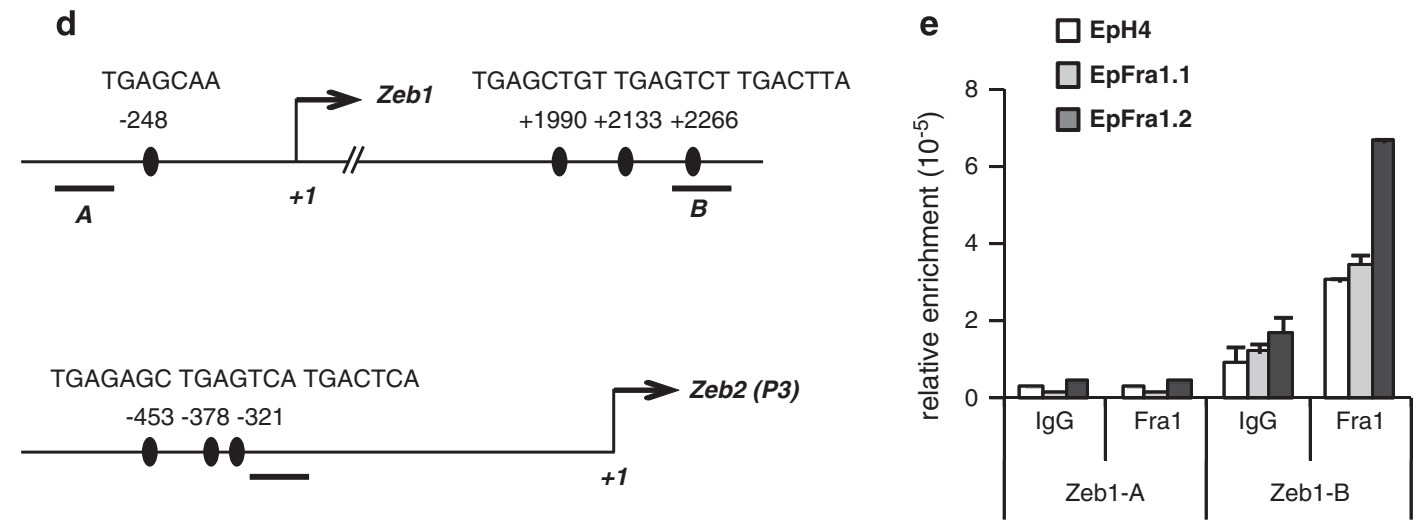

f

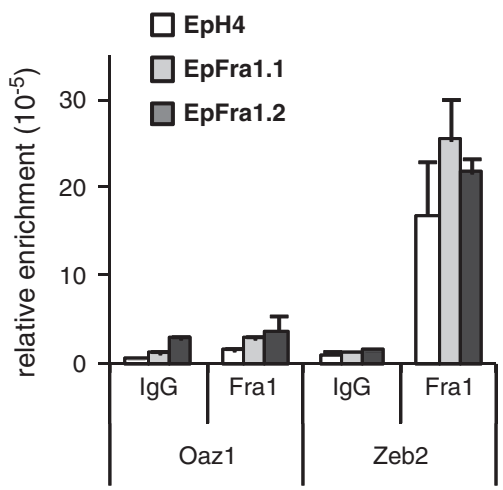

g

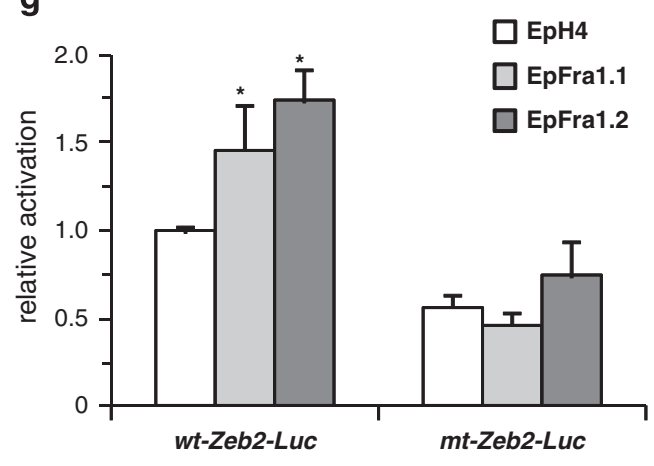

Figure 5 Increased Zeb1 and Zeb2 expression in EpFra1 cells. Analysis of (a) mRNA and (b) protein expression of E-box-binding proteins in EpH4-derived cells. Bars $=$ mean \pm S.D., $n=2$, expression in EpH4 is set to 1. Nuclear extracts were used to detect Zeb2 and Snail. Tubulin and Hdac3 are used to control loading for total and nuclear extracts, respectively. (c) qRT-PCR of Zeb1/2 targets genes in EpH4-derived cells. Bars = mean \pm S.D., $n=2$, expression in EpH4 is set to 1. (d) Scheme of the murine zeb1 and zeb2 promoters. Putative AP-1-binding sites and ChIP amplicons are depicted. ChIP analysis of (e) zeb1 and (f) zeb2 promoters in EpH4-derived cells using $\alpha$-Fra-1 or IgG. (e) Enrichment relative to input of the two zeb1 amplicons is compared. (f) A fragment in the Oaz1 (ornithine decarboxylase antizyme 1) coding region is used as an additional control for non-specific ChIP. Bars $=$ mean \pm S.D., $n=2$. (g) Reporter assay using wt and AP-1-mutant zeb2-luc constructs in EpH4-derived cells. Bars $=$ mean \pm S.D., wt-zeb2-luc: $n=6$ and ${ }^{*} P<0.05$, mut-zeb2-luc: $n=2$. Wt-reporter relative light unit values in $\mathrm{EpH} 4$ is set to 1 
a

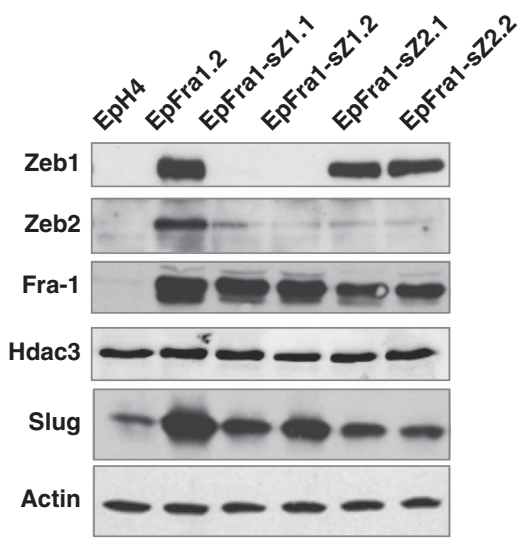

$\begin{array}{ll}\square \text { EpH4 } & \square \text { EpFra1-sZ1 } \\ \square \text { EpFra1.2 } & \text { EpFra1-sZ2 }\end{array}$
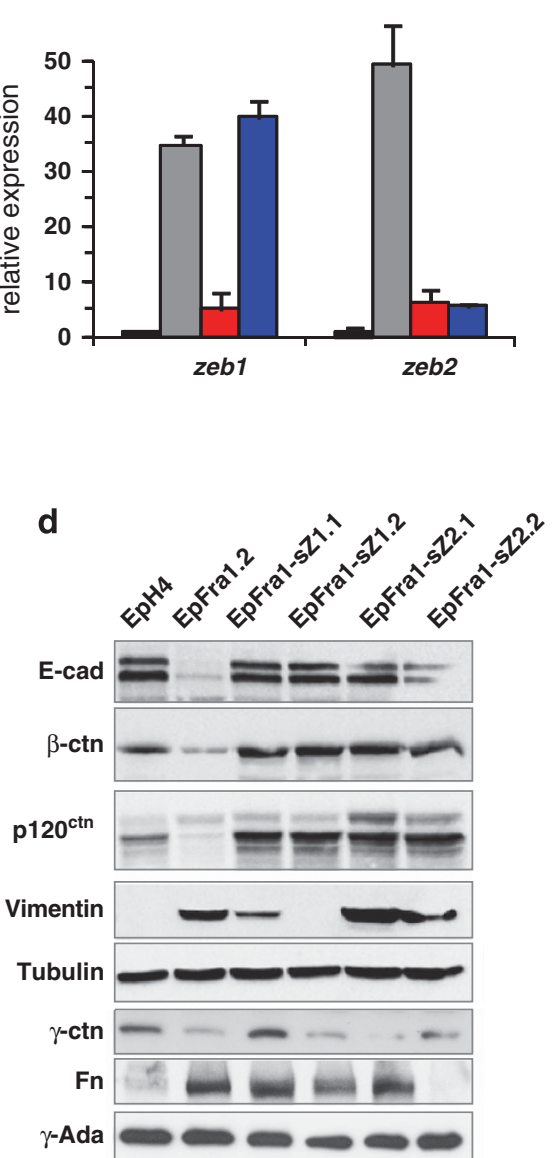

b

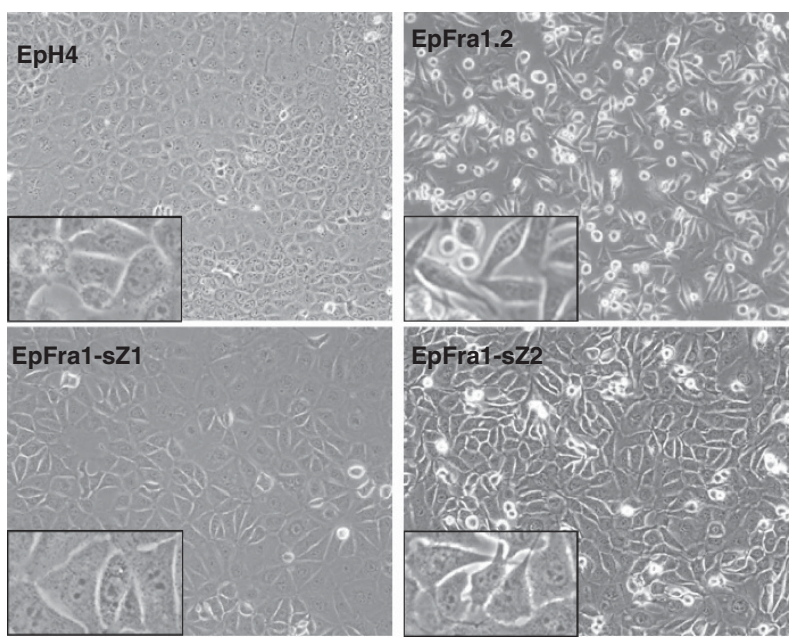

C

EpFra1-sZ1

$\square$ EpFra1.2 $\square$ EpFra1-sZ2

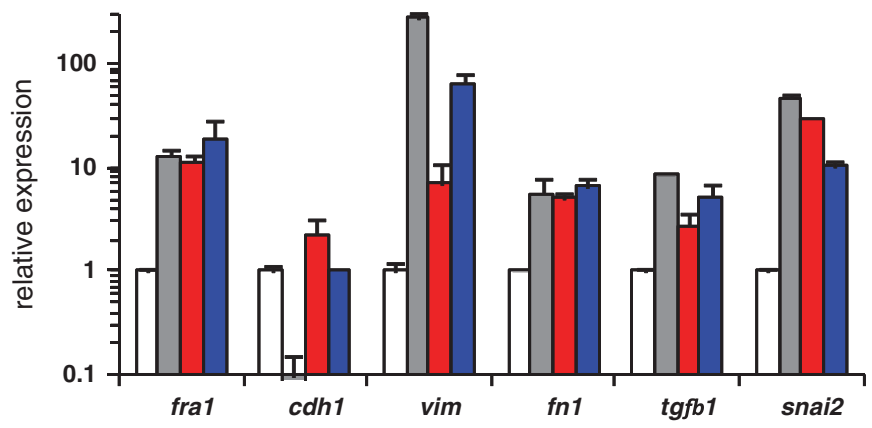

e

f
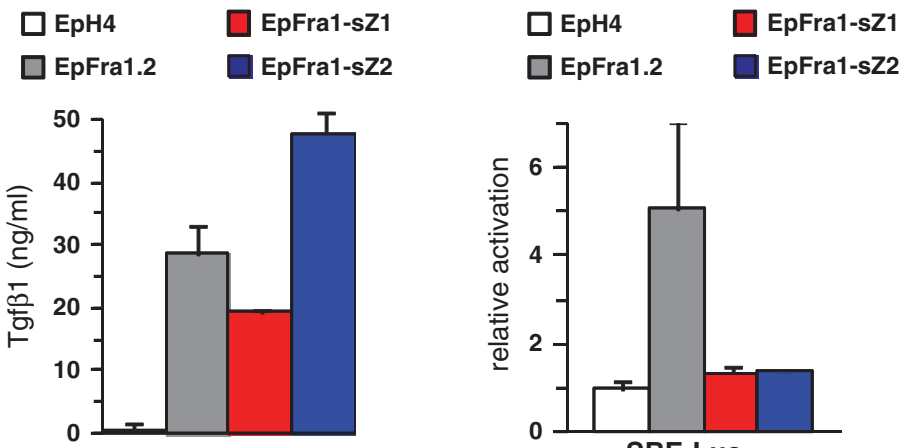

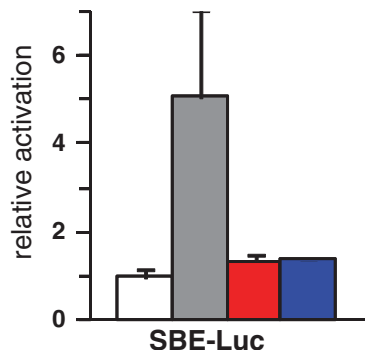

Figure 6 Knockdown of zeb1 or zeb2 in EpFra1 cells restores epithelial features. (a) Expression analysis of Zeb knockdown clones. Top: western blottiing. Note that nuclear extracts were used to detect Zeb2. Actin and Hdac3 are used to control loading for total and nuclear extracts, respectively. Bottom: qRT-PCR Bars $=$ mean \pm S.D., $n \geq 2$, expression in EpH4 is set to 1. (b) Representative phase-contrast photographs of parental and Zeb knockdown clones. (c) qRT-PCR analysis of Zeb knockdown clones. Bars $=$ mean \pm S.D., $n \geq 2$, expression in EpH4 is set to 1. (d) Western blotting analysis of epithelial and mesenchymal proteins. Tubulin and $\gamma$-Adaptin are used to control loading. (e) TGF $\beta 1$ ELISA in the conditioned medium and (f) relative Smad-reporter activity in Zeb knockdown clones. Bars $=$ mean \pm S.D., $n=2$, relative light units in EpH4 is set to 1 
these being Zeb1/2 targets (Figure $6 \mathrm{c}$ and Supplementary Figures S10a-d). Interestingly, only few mesenchymal markers, such as vimentin, mmp2, mmp10 and the a5 Integrin were decreased in Zeb1 and/or Zeb2 knockdown cells (Figure $6 c$ and Supplementary Figures S10c and d). Western blotting confirmed E-cadherin, $\beta$-catenin and p120catenin restoration and decreased Vimentin in EpFra1-sZ1 cells (Figure 6d). Importantly, while Tfg $\beta 1$ mRNA and protein were not affected by Zeb1/2 knockdown (Figures $6 c$ and e), Smad2/3 reporter assays revealed decreased TGF $\beta$ pathway activity (Figure 6f). This is consistent with the reported crosstalk between Zeb and Smad proteins on Smadresponsive promoters. ${ }^{41}$ Reduced snai2/Slug was also observed upon Zeb1/2 knockdown (Figures 6a and c), likely due to decreased TGF $\beta$ pathway activity. These data indicate that decreasing Zeb1 and/or Zeb2 in EpFra1 cells restored a significant part of the epithelial programme.

\section{Targeting Zeb1 and Zeb2 in EpFra1 cells modulates} tumorigenesis. EpFra1-sZ1 and EpFra1-sZ2 presented similar proliferation characteristics to EpFra1 cells, with slightly lower cell counts in long-term EpFra1-sZ1 cultures (Figure 7a). In addition, EpFra1-sZ1 but not EpFra1-sZ2 cells displayed decreased motility and invasiveness in Transwell and Matrigel invasion assays (Figure 7b). Furthermore, EpFra1-sZ1 and EpFra1-sZ2 cells produced smaller mammary tumours, but overall Ki67-index was comparable (Figure 7c). Unlike the parental EpFra1 cells, EpFra1-sZ1 and EpFra1-sZ2 tumours stained positive for E-cadherin (Figure 7d). Interestingly, while EpFra1-sZ1 and EpFra1-sZ2 cells were still able to colonize the lungs, tumours deriving from EpFra1-sZ1 cells retained E-cadherin expression, whereas those deriving from EpFra1-sZ2 cells did not (Figure 7e). Furthermore, unlike EpFra1 and EpFra1-sZ2derived tumours, EpFra1-sZ1-derived tumours were largely negative for Vimentin (Figure 7e). IHC confirmed that Fra-1 expression was maintained in transplanted EpFra1 cells while Zeb-1 was decreased in EpFra1-sZ1 tumours (Figure 7e).

In conclusion, our data support a model by which Fra-1 induces EMT in mouse mammary epithelial cells by modulating EMT-TF and TGF $\beta$ signalling. Increased tgfb1, zeb1 and zeb2 expression is at least partly due to direct promoter binding and Zeb1/2 are essential for Fra-1-induced EMT. Fra-1 also increases snai2/Slug, likely through Zeb1/2 and TGF $\beta$ signalling. Increased EMT-TF and TGF $\beta$ signalling decreases E-cadherin and epithelial gene expression, whereas mesenchymal genes are increased, ultimately leading to the acquisition of invasive, tumorigenic and metastatic properties (Figure 7f).

\section{Discussion}

GEMMs are of great potential for therapy development, in particular when tackling the metastatic, systemic phase of cancer. However, a better knowledge of the evolutionary conservation of the cellular and molecular interplays from studies in human tumour material is a critical prerequisite to using preclinical GEMMs. Several reports have connected Fra-1/AP-1 in human breast cancer to increased expression of the EMT-TFs Zeb1 and Zeb2 and to a lesser extent
Slug. ${ }^{12-15,17}$ We investigated whether Fra-1/AP-1 and the EMT-TFs also correlated in mouse mammary EMT. Expression of Fra-1 in EpH4 cells induced EMT and increased proliferation, migration and invasiveness. Unlike proliferation, the EMT phenotype was largely dependent on increased Zeb1/2 expression, and despite a deregulation of cell deathrelated genes, cell survival was not overtly affected. Importantly, Fra-1-expressing cells grew as orthotopic tumours and colonized the lungs following vascular delivery. Our findings are consistent with reports that Fra-1 affects the proliferation and motility of human breast cancer cell lines ${ }^{17,42}$ and that Fra1 is a critical EMT effector downstream of Ras signalling. ${ }^{13,14}$

Ras and TGF $\beta$ cooperate to induce and stabilize a mesenchymal switch in $\mathrm{EpH} 4$ cells. ${ }^{21,22,40}$ Unlike human breast cancer cells where upstream signalling kinases activation is required, ${ }^{14,16}$ ectopic Fra-1 expression was sufficient for EMT and tumorigenesis in EpH4 cells. Moreover, Fra-1 increased TGF $\beta$ expression and production, similarly to c-Fos and unlike oncogenic Ras, ${ }^{43}$ thus TGF $\beta$ treatment was not necessary for EMT in EpFra1 cells. We demonstrated that Fra-1 binds to two regions in the murine tgfb1 promoter containing several AP-1-binding elements. As autocrine TGF $\beta$ production has also been observed in human breast cancer cells, where extracellular signal-regulated kinase 2 (ERK2)induced EMT depends on Fra- $1^{14}$ and as Fra-1 binds the human TGFB2 gene in colorectal cancer cells, ${ }^{44}$ it is tempting to speculate that Fra-1/AP-1 modulates the transcription of TGFB genes in the context of EMT. However, inhibition of TGF $\beta$ signalling in EpFra1 cells only partially restored epithelial markers expression, similarly to the situation during Fos- or ERK2-induced EMT. ${ }^{14,43}$ Thus, TGF $\beta$ signalling is likely insufficient for EMT in the context of ectopic Fra-1 expression.

The hallmark of Fra-1-induced EMT is E-cadherin downregulation. Several mechanisms modulating E-cadherin expression have been described. Ectopic c-Fos expression in mouse mammary adenocarcinoma cells was correlated with E-cadherin repression by promoter hypermethylation. ${ }^{45}$ As treatment of EpFra1 cells with DNA methylation or histone acetylation inhibitors failed to restore E-cadherin (data not shown), and given the published data using human cancer cells, it seemed conceivable that downregulation of E-cadherin by Fra-1 was mediated by EMT-TFs. Zeb1, Zeb2 and Slug were increased in EpFra1 cells and the expression of several bona fide Zeb1/2 target genes altered. Furthermore, expression profiling revealed a significant overlap between the gene sets affected by Fra- 1 in $\mathrm{EpH} 4$ cells and data sets from human breast cancer cell lines, in which Zeb1 or Zeb2 expression had been targeted. ${ }^{34,35}$ Consistent with the data from human cells, ${ }^{14}$ RNA interference demonstrated that reducing the expression of Zeb1 and Zeb2, and to a lesser extent Zeb2 in EpFra1 cells, restored the expression of E-cadherin and most Zeb target genes and reduced migration and invasion in vitro. Interestingly, Zeb2 was decreased in Zeb1 knockdown cells and increased upon ectopic Zeb1 expression. Although we cannot formally exclude off-target effects from the Zeb1 shRNA or an indirect consequence of the EMT/mesenchymal-to-epithelial transition phenotype, this indicates that Zeb1 participates in the control of Zeb2 expression in mouse mammary cells as documented in 


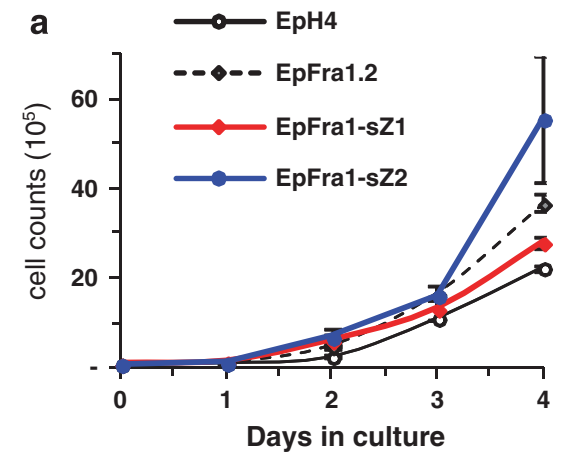

C

$\square$ EpFra1.2

EpFra1-sZ1

EpFra1-sZ2
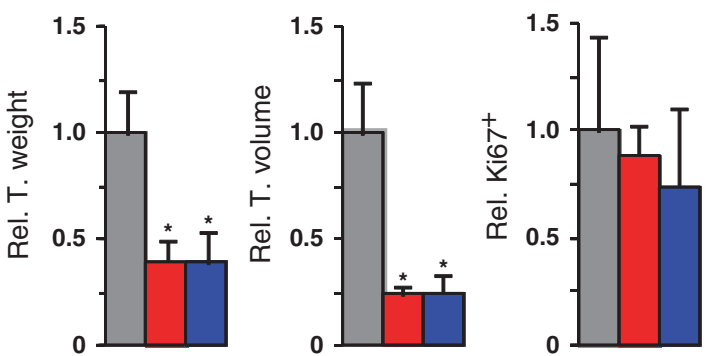

e

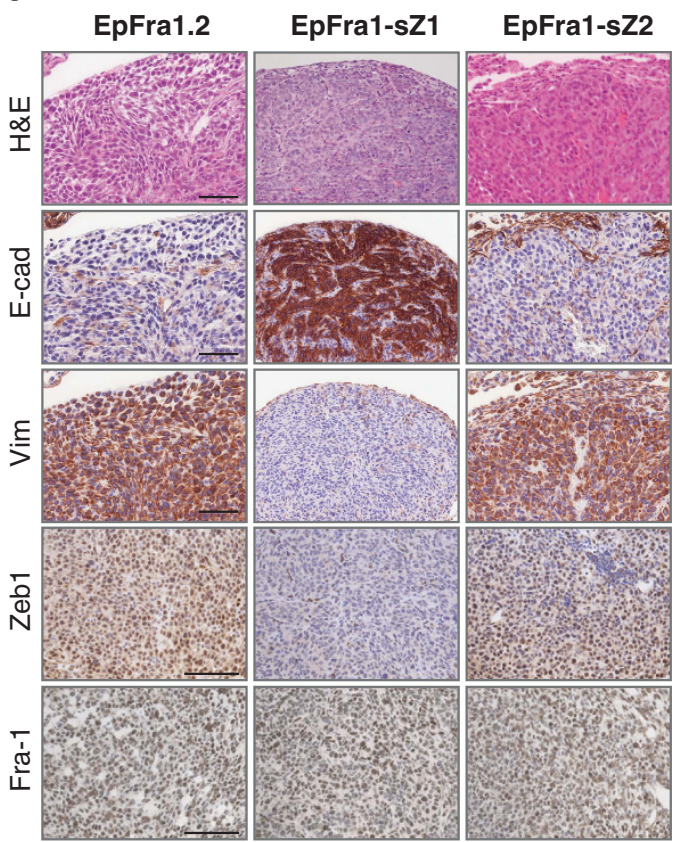

b
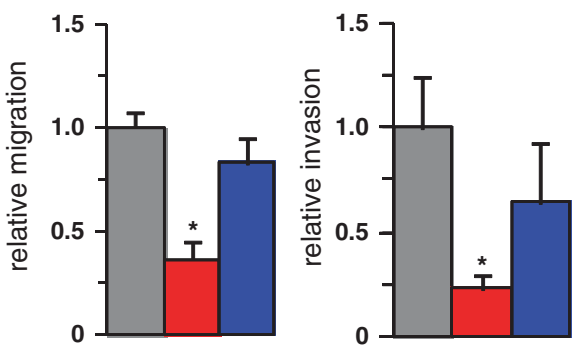

d

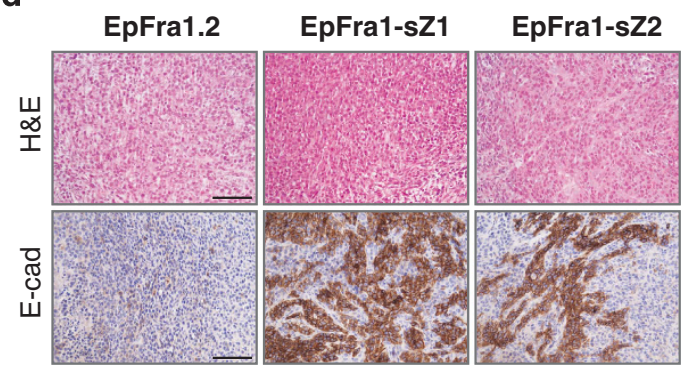

f

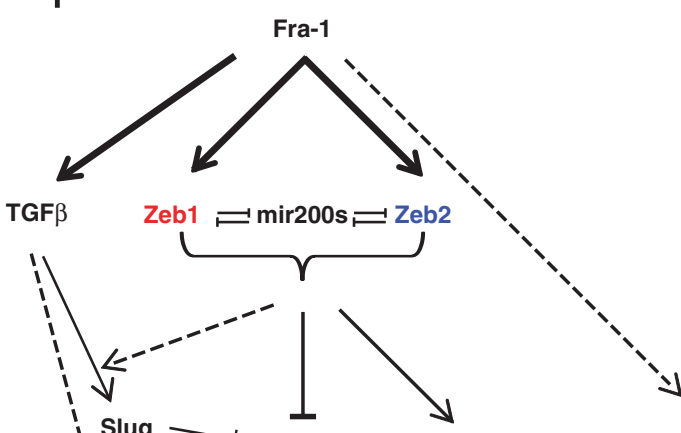

Slug E-cad mesenchymal genes

\&

epithelial genes
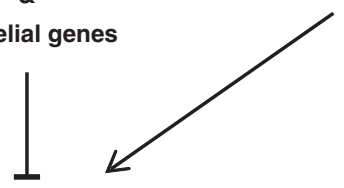

EMT, invasion

\& metastasis

Figure 7 Effects of zeb1 or zeb2 knockdown in vitro and in xenograft models. (a) Proliferation, (b) migration and invasion of Zeb knockdown clones. (c) Quantification of tumour volume, weight $(n=3)$ and Ki67 IHC $(n=2)$ relative to EpFra1 cells (d) H\&E and E-cadherin IHC of EpFra1 or Zeb knockdown mammary gland tumour sections. $\mathrm{Bar}=100 \mu \mathrm{M}$. Bars $=$ mean \pm S.D. ${ }^{*} P<0.05$. (e) H\&E, E-cadherin, Vimentin, Zeb1 and Fra-1 IHC on lung sections from EpFra1 or Zeb knockdown tail vein injections. Bar $=100 \mu \mathrm{M}$. (f) Schematic model of the function of Fra-1 in EMT. In EpH4 cells, Fra-1 increases tgfb1, zeb1 and zeb2 expression by direct promoter binding. Fra-1 increases snai2/Slug, likely through modulating Zeb expression and TGF $\beta$ signalling. Increased E-box-binding TFs and TGF $\beta$ signalling decreases E-cadherin and epithelial gene expression, while mesenchymal genes are increased, ultimately leading to the acquisition of invasive, tumorigenic and metastatic properties 
human melanoma. ${ }^{46}$ Transplantation experiments indicated that EpFra1 cells with Zeb1/2 knockdown displayed decreased orthotopic tumorigenesis, but most strikingly the cells could still colonize the lungs, with a notable fraction of the Zeb1 knockdown tumours retaining E-cadherin expression. Thus Zeb1 and Zeb2 are the critical mediators of E-cadherin downregulation and EMT downstream of Fra-1, while other targets of Fra-1, independent of Zeb1/2, might sustain the proliferative and tumorigenic capacity of EpFra1 cells in vivo.

TGF $\beta$ signalling, miR-200 and miR-221/222/Trps1 appeared insufficient to explain increased Zeb1 and Zeb2 expression in EpFra1 cells. We evaluated whether Fra-1 directly regulates Zeb1 and Zeb2 transcription, focussing on genomic sequences with putative AP-1-binding elements conserved in the human genes. Fra-1 bound the P3 promoter of Zeb2, and subsequent reporter assays demonstrated that Fra-1 activation of a P3 promoter fragment depends on three novel AP-1 sites. To our knowledge, this is the first time that a direct transcriptional regulation of Zeb2 by AP-1 is reported. In the human ZEB1 gene, two Fra-1/AP-1-binding regions have been reported by ChIP in breast ${ }^{38}$ and colorectal ${ }^{44}$ cancer cells. We show that Fra-1 binds a region of the mouse Zeb1 gene homologous to the recently identified genomic determinant of human breast cancer cell plasticity. ${ }^{25}$ Whether Fra-1 regulates ZEB1 expression in human breast cancer cells by altering the dynamic of histone modifications in this region is an attractive possibility that merits further investigation. In conclusion, our work provides novel insights into how Fra-1/ AP-1 is connected to the complex network of EMT modulators.

\begin{abstract}
Materials and Methods
Cell lines. The origin, cultivation and properties of EpH4, EpRas and EpRasXT and EpGFP and EpdEF1GFP cells have been previously described. ${ }^{22,40,47}$ NMuMG cells and cultivation conditions were obtained from ATCC (Barcelona, Spain). EpFra1, EpRas NMuMG-GFP and NMuMG-Fra1ER cells were generated by retroviral gene transfer as described in Bakiri et al., ${ }^{48}$ using pBabeFra1, pBabeGFP or pBabeFra1ER retroviral plasmids. Several clones expressing Fra-1 were isolated, and two representatives were used for further analysis. To knock down Fra-1 in EpFra1 cells, pLKO.1-based lentiviral particles expressing sequence-verified Fra-1silencing shRNA (Supplementary Table S2) were used, and the pools of infected cells were assayed. To knock down either Zeb1 or Zeb2 in EpFra1 cells, lentiviral particles containing five different validated shRNAs for each target were used (Supplementary Table S2), and stable pools were generated using G418. For inhibition of TGF $\beta 1$ signalling, cells were treated with $20 \mu \mathrm{M}$ TGF $\beta$-receptor 1 inhibitor (Sigma, Madrid, Spain) for the indicated time periods.
\end{abstract}

Immunofluorescence. Cells grown on $0.4-\mu \mathrm{m}$ filters (BD, Schwechat, Austria) were fixed in $4 \%$ PFA/PBS for $15 \mathrm{~min}$ and postfixed with ice-cold Methanol for 5 min. Cells were permeabilized with PBS $0.1 \%$ Triton X-100 for 2 min and incubated for $1 \mathrm{~h} 30 \mathrm{~min}$ in a humid chamber with AlexaFluor 488-Phalloidin (Invitrogen, Barcelona, Spain) or with the following primary antibodies: E-cadherin, $\alpha$-catenin, $\beta$-catenin, $\gamma$-catenin and $120^{\mathrm{ctn}}$, Fibronectin, and Fra- 1 (Supplementary Table S2), all at $1: 250$ in $0.5 \%$ BSA/PBS. Cells were washed with PBS and incubated with the appropriate AlexaFluor 488 (Invitrogen) or DyLight 549 (Pierce, Vienna, Austria) secondary antibody at $1: 250$ in $0.5 \%$ BSA/PBS for $1 \mathrm{~h}$. Filters were washed twice with PBS and mounted onto microscope glass slides using Vectashield-DAPI (Vector Laboratories, Vienna, Austria). Imaging was performed on a Zeiss Axioplan 2 microscope equipped with a CoolSnap HQ camera (Photometrics, Frankfurt, Germany).

Proliferation, migration and invasion assays. In all, $5 \times 10^{4}$ cells were seeded into each well of a 24-well tissue culture plate. Cells were counted in triplicate wells, on consecutive days until confluence. For Transwell migration, $1 \times 10^{5}$ overnight serum-starved cells were seeded in duplicates in DMEM/F12 with $0.1 \%$ BSA onto a 8- $\mu \mathrm{m}$ Filter (BD) in 6-well plates. Medium supplemented with 10\% FBS was provided below the filters as chemoattractant. Migrated cells were stained with Haematoxylin and counted 3 days later. Invasion assays were carried in triplicates in Matrigel Invasion chambers (BD) exactly as described in the manufacturer's protocol with medium supplemented with 10\% FBS as a chemoattractant. Invading cells were stained with Haematoxylin and counted 3 days later.

Xenografts. Six-to-eight-weeks-old female NMRI ${ }^{\text {nu/nu }}$ mice were used for all xenograft experiments. Mammary gland xenografts were performed by injecting $1 \times 10^{5}$ cells in $25 \mu \mathrm{l}$ PBS into the second and fourth mammary glands. For lung colonization assays, $1,5 \times 10^{5}$ cells in $100 \mu$ l PBS were injected into the lateral tail vein. Animals' health and mammary tumour growth were monitored daily, and mice were killed between 14 and 21 days after injection. All mouse experiments were performed in accordance with the local and institutional regulations.

Histological analysis. Tissues were fixed in $4 \%$ formalin and embedded in paraffin, and 4- $\mu \mathrm{m}$ sections were prepared. $\mathrm{IHC}$ was performed on deparaffinized slides using antibodies against Fra-1, Ki67, E-cadherin, Vimentin and Zeb-1 (Supplementary Table S2) and the appropriate VECTASTAIN Elite ABC Kit (Vector Laboratories). Haematoxylin was used for nuclear counterstaining.

RNA and protein analyses. RNA was isolated with Trizol (Sigma), and complementary DNA was synthesized with Ready-To-Go-You-Prime-First-Strand Beads (GE Healthcare, Madrid, Spain). Primer pairs were designed using the Primer-BLAST online programme from NCBI, and sequences are provided in Supplementary Table S3. Quantitative PCR were performed using GoTaq 9PCR Master Mix (Promega, Madrid, Spain) and an Eppendorf fluorescence thermocycler (Eppendorf, Madrid, Spain). The comparative cycle threshold method was used for quantification. Expression levels were normalized using at least one housekeeping gene (hprt, gapdh, rp/4, rps29). For protein extraction, nuclei were prepared by incubating the cells $15 \mathrm{~min}$ on ice in hypotonic buffer (10 mM HEPES pH 7.9; 10 mM KCl; 0.1 mM EDTA; 0.1 mM EGTA; 1 mM DTT; $0.5 \mathrm{mM}$ PMSF and $1 \times$ complete protease inhibitor cocktail (Roche, Madrid, Spain). Cells or nuclei were lysed in ice-cold lysis buffer $(50 \mathrm{mM}$ Tris- $\mathrm{HCl} \mathrm{pH} \mathrm{7.4;} 150 \mathrm{mM}$ $\mathrm{NaCl} ; 1 \mathrm{mM}$ EDTA; 1\% NP-40; 1\% Sodium-deoxycholate; 1 mM Na3VO4; $25 \mathrm{mM} \mathrm{NaF}$; $1 \mathrm{mM}$ PMSF; $1 \times$ complete protease inhibitor cocktail (Roche); $1 \% 1 \mathrm{M} \mathrm{DTT}$ and $1 \% \beta$ glycerol phosphate). Protein concentration was assessed using the BCA kit (Pierce), and the nuclear extracts' quality was first controlled using western blotting for SP1, HP1 $\alpha$, Hdac3 and Gapdh as exemplified in Supplementary Figures. Western blottings were performed as described ${ }^{49,50}$ using the primary antibodies listed in Supplementary Table S2. Blots were incubated with horseradish peroxidase-coupled secondary antibodies (GE Healthcare) and developed using Amersham ECL (GE Healthcare) or Luminata (Pierce) and ECL Hyperfilms (GE Healthcare) or a Chemidoc device (Bio-Rad, Madrid, Spain).

Chromatin immunoprecipitation. ChIP was performed as previously described ${ }^{51}$ using Fra-1 antibody (Santa Cruz, Madrid, Spain) or rabbit lgG. qPCR was used to monitor amplified fragments, and the comparative cycle threshold method was used for quantification. Primer sequences are provided in Supplementary Table S3. For each cell line, values were expressed as the percentage of amplification of input chromatin.

ELISA. TGF $\beta 1$ was measured in culture supernatants from triplicate cultures with comparable cell numbers using the TGF $\beta 1$ ELISA kit (R\&D systems, Madrid, Spain) according to the manufacturer's instructions.

Reporter assay. A 500-bp fragment of the mouse Zeb2 P3 promoter was amplified by high-fidelity PCR and cloned upstream of the firfly Luciferase in the pGL4.10 vector (Promega). The three potential AP-1-binding sites in the reporter were mutated to TGAattC, gagCTCC and TctAGaCA, respectively using the Quickchange mutagenesis kit (Stratagene, Madrid, Spain) according to the manufacturer's instructions. For the reporter assays, the different cell lines were cultivated in six-well plates and transiently transfected (Lipofectamin 2000, Life Technologies, Vienna, Austria) with Smad2 or Smad3 reporter ${ }^{52}$ or wild-type/mutant Zeb2 promoter reporter constructs and the phRG-Renilla construct (Promega) for normalization. Luciferase activity was assayed $40 \mathrm{~h}$ later using a Dual Luciferase kit (Invitrogen or Promega) according to the manufacturer's instructions.

Gene expression profiling. cDNA was generated from $3 \mu \mathrm{g}$ of total RNA using Superscriptll, purified (Qiagen, Hilden, Germany) and labelled with 
fluorescent dyes (Alexa 555 and Alexa 647 , Invitrogen) and hybridized at $50^{\circ} \mathrm{C}$ overnight in triplicates on microarray slides. In-house printed oligonucleotide slides containing $>22500$ oligonucliotide sequences were used. Hybridized slides were scanned on a fluorescent scanner Axon 4000B (Molecular Devices GmbH, Biberach an der Riss, Germany), and the resulting images were processed for spot and local background identification using the GenePix Pro 6.0 software (Molecular Devices $G \mathrm{mbH})$. Background correction and normalization was performed using $R$ and the Limma package from Bioconductor (Fred Hutchinson Cancer Research Center, Seattle, WA, USA). Moderated $t$-statistic was computed for each probe and for each contrast, and an analysis of variance test was applied to calculate $P$-values. Only annotated genes showing at least two-fold change in expression in both EpFra1 clones relative to the parental $\mathrm{EpH} 4$, and a $P$-value $<0.05$ were considered. Zeb and AP-1 target genes were identified using tools from the GSEA website developed at the Broad Institute. Gene expression profiling of EpRas and two EpRas-Fra1 clones was performed in a similar way, with a focus on Zeb1/2 target genes. The EpFra1 gene expression profiling data have been deposited in NCBl's Gene Expression Omnibus and are accessible through GEO Series accession number GSE56089 at http://www.ncbi.nlm.nih.gov/geo/query/acc.cgi?acc = GSE56089.

Statistical analyses. Unless otherwise specified, data in plots and bar graphs are presented as mean \pm S.D. Statistical analysis was performed using nondirectional two-tailed Student's $t$-test. $P<0.05$ was considered significant.

Acknowledgements. We are very grateful to Dr. M Perez-Moreno, Dr. V Sex and Dr. G Tebb for critical reading of the manuscript, Dr. K Matsuo, Dr. V OrianRousseau and Dr. MA Quintela for suggestions and sharing reagents, $\mathrm{H}$ Scheuch for help with the microarray analysis and $\mathrm{V}$ Bermeo and $\mathrm{C}$ Blanco for technical assistance. The IMP is funded by Boehringer Ingelheim. Research at the University of Veterinary Medicine, Vienna is supported by the Austrian Science Fund (FWF: SFB F28). SCH was the recipient of a Boehringer Ingelheim Fonds PhD fellowship. Research in EFW's group at the CNIO is supported by the Banco Bilbao Vizcaya Argentaria Foundation (F-BBVA), the Spanish Ministry of Economy (Grant BFU201240230) and a European Research Council-advanced grant (ERC-FCK/2008/37).

1. Kalluri R, Weinberg RA. The basics of epithelial-mesenchymal transition. J Clin Invest 2009; 119: $1420-1428$

2. Savagner P. Leaving the neighborhood: molecular mechanisms involved during epithelial-mesenchymal transition. Bioessays 2001; 23: 912-923.

3. Lamouille S, Xu J, Derynck R. Molecular mechanisms of epithelial-mesenchymal transition. Nat Rev Mol Cell Biol 2014; 15: 178-196.

4. Thiery JP, Acloque H, Huang RY, Nieto MA. Epithelial-mesenchymal transitions in development and disease. Cell 2009; 139: 871-890.

5. Tsai JH, Yang J. Epithelial-mesenchymal plasticity in carcinoma metastasis. Genes Dev 2013; 27: 2192-2206.

6. Peinado H, Olmeda D, Cano A. Snail, Zeb and bHLH factors in tumour progression: an alliance against the epithelial phenotype? Nat Rev Cancer 2007; 7: 415-428.

7. Tam WL, Weinberg RA. The epigenetics of epithelial-mesenchymal plasticity in cancer. Nat Med 2013; 19: 1438-1449

8. Tse JC, Kalluri R. Mechanisms of metastasis: epithelial-to-mesenchymal transition and contribution of tumor microenvironment. J Cell Biochem 2007; 101: 816-829.

9. Lamouille S, Subramanyam D, Blelloch R, Derynck R. Regulation of epithelial-mesenchymal and mesenchymal-epithelial transitions by microRNAs. Curr Opin Cell Biol 2013; 25 200-207.

10. Reisfeld RA. The tumor microenvironment: a target for combination therapy of breast cancer. Crit Rev Oncog 2013; 18(1-2): 115-133

11. Verde P, Casalino L, Talotta F, Yaniv M, Weitzman JB. Deciphering AP-1 function in tumorigenesis: fra-ternizing on target promoters. Cell Cycle 2007; 6: 2633-2639.

12. Desmet CJ, Gallenne T, Prieur A, Reyal F, Visser NL, Wittner BS et al. Identification of a pharmacologically tractable Fra-1/ADORA2B axis promoting breast cancer metastasis. Proc Natl Acad Sci USA 2013; 110: 5139-5144.

13. Tam WL, Lu H, Buikhuisen J, Soh BS, Lim E, Reinhardt F et al. Protein kinase $C$ alpha is a central signaling node and therapeutic target for breast cancer stem cells. Cancer Cell 2013; 24: $347-364$

14. Shin S, Dimitri CA, Yoon SO, Dowdle W, Blenis J. ERK2 but not ERK1 induces epithelialto-mesenchymal transformation via DEF motif-dependent signaling events. Mol Cell 2010; 38: 114-127.

15. Stinson S, Lackner MR, Adai AT, Yu N, Kim HJ, O'Brien C et al. TRPS1 targeting by miR-221/222 promotes the epithelial-to-mesenchymal transition in breast cancer. Sci Signal 2012; 4 ra41.
16. Belguise K, Milord S, Galtier F, Moquet-Torcy G, Piechaczyk M, Chalbos D. The PKCtheta pathway participates in the aberrant accumulation of Fra- 1 protein in invasive ER-negative breast cancer cells. Oncogene 2012; 31: 4889-4897.

17. Chen H, Zhu G, Li Y, Padia RN, Dong Z, Pan ZK et al. Extracellular signal-regulated kinase signaling pathway regulates breast cancer cell migration by maintaining slug expression. Cancer Res 2009; 69: 9228-9235.

18. Reichmann E, Schwarz H, Deiner EM, Leitner I, Eilers M, Berger J et al. Activation of an inducible c-FosER fusion protein causes loss of epithelial polarity and triggers epithelial-fibroblastoid cell conversion. Cell 1992; 71: 1103-1116.

19. Grunert S, Jechlinger M, Beug H. Diverse cellular and molecular mechanisms contribute to epithelial plasticity and metastasis. Nat Rev Mol Cell Biol 2003; 4: 657-665.

20. Janda E, Lehmann K, Killisch I, Jechlinger M, Herzig M, Downward J et al. Ras and TGF [beta] cooperatively regulate epithelial cell plasticity and metastasis: dissection of Ras signaling pathways. J Cell Biol 2002; 156: 299-313.

21. Jechlinger $\mathrm{M}$, Grunert S, Tamir IH, Janda $\mathrm{E}$, Ludemann $\mathrm{S}$, Waerner $\mathrm{T}$ et al. Expression profiling of epithelial plasticity in tumor progression. Oncogene 2003; 22: 7155-7169.

22. Oft M, Peli J, Rudaz C, Schwarz H, Beug H, Reichmann E. TGF-beta1 and Ha-Ras collaborate in modulating the phenotypic plasticity and invasiveness of epithelial tumor cells. Genes Dev 1996; 10: 2462-2477.

23. Fialka I, Schwarz H, Reichmann E, Oft M, Busslinger M, Beug H. The estrogen-dependent c-JunER protein causes a reversible loss of mammary epithelial cell polarity involving a destabilization of adherens junctions. J Cell Biol 1996; 132: 1115-1132.

24. Gyorffy B, Lanczky A, Eklund AC, Denkert C, Budczies J, Li $Q$ et al. An online survival analysis tool to rapidly assess the effect of 22,277 genes on breast cancer prognosis using microarray data of 1,809 patients. Breast Cancer Res Treat 2010; 123: 725-731.

25. Chaffer CL, Marjanovic ND, Lee T, Bell G, Kleer CG, Reinhardt F et al. Poised chromatin at the ZEB1 promoter enables breast cancer cell plasticity and enhances tumorigenicity. Cell 2013; 154: 61-74.

26. Subramanian A, Tamayo P, Mootha VK, Mukherjee S, Ebert BL, Gillette MA et al. Gene set enrichment analysis: a knowledge-based approach for interpreting genome-wide expression profiles. Proc Natl Acad Sci USA 2005; 102: 15545-15550.

27. Onder TT, Gupta PB, Mani SA, Yang J, Lander ES, Weinberg RA. Loss of E-cadherin promotes metastasis via multiple downstream transcriptional pathways. Cancer Res 2008; 68: $3645-3654$

28. Takebe N, Warren $R Q$, Ivy SP. Breast cancer growth and metastasis: interplay between cancer stem cells, embryonic signaling pathways and epithelial-to-mesenchymal transition. Breast Cancer Res 2011; 13: 211

29. Lei W, Rushton JJ, Davis LM, Liu F, Ness SA. Positive and negative determinants of target gene specificity in myb transcription factors. J Biol Chem 2004; 279: 29519-29527.

30. Koinuma D, Tsutsumi S, Kamimura N, Taniguchi $\mathrm{H}$, Miyazawa $\mathrm{K}$, Sunamura $\mathrm{M}$ et al. Chromatin immunoprecipitation on microarray analysis of Smad2/3 binding sites reveals roles of ETS1 and TFAP2A in transforming growth factor beta signaling. Mol Cell Biol 2009; 29: $172-186$.

31. Cesi V, Casciati A, Sesti F, Tanno B, Calabretta B, Raschella G. TGFbeta-induced c-Myb affects the expression of EMT-associated genes and promotes invasion of ER+ breast cancer cells. Cell Cycle 2011; 10: 4149-4161.

32. Valcourt U, Kowanetz M, Niimi H, Heldin CH, Moustakas A. TGF-beta and the Smad signaling pathway support transcriptomic reprogramming during epithelial-mesenchymal cell transition. Mol Biol Cell 2005; 16: 1987-2002.

33. Geiser AG, Kim SJ, Roberts AB, Sporn MB. Characterization of the mouse transforming growth factor-beta 1 promoter and activation by the Ha-ras oncogene. Mol Cell Biol 1991; 11: 84-92.

34. Aigner K, Dampier B, Descovich L, Mikula M, Sultan A, Schreiber M et al. The transcription factor ZEB1 (deltaEF1) promotes tumour cell dedifferentiation by repressing master regulators of epithelial polarity. Oncogene 2007; 26: 6979-6988.

35. Mejlvang J, Kriajevska M, Vandewalle C, Chernova T, Sayan AE, Berx G et al. Direct repression of cyclin D1 by SIP1 attenuates cell cycle progression in cells undergoing an epithelial mesenchymal transition. Mol Biol Cell 2007; 18: 4615-4624.

36. D'Amato NC, Howe EN, Richer JK. MicroRNA regulation of epithelial plasticity in cancer. Cancer Lett 2013; 341: 46-55.

37. Lambertini E, Lolli A, Vezzali F, Penolazzi L, Gambari R, Piva R. Correlation between Slug transcription factor and miR-221 in MDA-MB-231 breast cancer cells. BMC Cancer 2012; 12. 445

38. Yang S, Du J, Wang Z, Yan J, Yuan W, Zhang J et al. Dual mechanism of deltaEF1 expression regulated by bone morphogenetic protein-6 in breast cancer. Int $J$ Biochem Cell Biol 2009; 41: 853-861.

39. Nelles L, Van de Putte T, van Grunsven L, Huylebroeck D, Verschueren K. Organization of the mouse Zfhx1b gene encoding the two-handed zinc finger repressor Smad-interacting protein-1. Genomics 2003; 82: 460-469.

40. Eger A, Aigner K, Sonderegger S, Dampier B, Oehler S, Schreiber M et al. DeltaEF1 is a transcriptional repressor of $\mathrm{E}$-cadherin and regulates epithelial plasticity in breast cancer cells. Oncogene 2005; 24: 2375-2385.

41. Fuxe J, Vincent T, Garcia de Herreros A. Transcriptional crosstalk between TGF-beta and stem cell pathways in tumor cell invasion: role of EMT promoting Smad complexes. Cell Cycle 2010; 9: 2363-2374.

42. Belguise K, Kersual N, Galtier F, Chalbos D. FRA-1 expression level requlates proliferation and invasiveness of breast cancer cells. Oncogene 2005; 24: 1434-1444. 
43. Eger A, Stockinger A, Park J, Langkopf E, Mikula M, Gotzmann J et al. beta-Catenin and TGFbeta signalling cooperate to maintain a mesenchymal phenotype after FosER-induced epithelial to mesenchymal transition. Oncogene 2004; 23: 2672-2680.

44. Diesch J, Sanij E, Gilan O, Love C, Tran H, Fleming NI et al. Widespread FRA1-dependent control of mesenchymal transdifferentiation programs in colorectal cancer cells. PLoS One 2014; 9: e88950.

45. Mejlvang J, Kriajevska M, Berditchevski F, Bronstein I, Lukanidin EM, Pringle JH et al. Characterization of E-cadherin-dependent and -independent events in a new model of c-Fosmediated epithelial-mesenchymal transition. Exp Cell Res 2007; 313: 380-393.

46. Caramel J, Papadogeorgakis E, Hill L, Browne GJ, Richard G, Wierinckx A et al. A switch in the expression of embryonic EMT-inducers drives the development of malignant melanoma. Cancer Cell 2013; 24: 466-480.

47. Reichmann E, Ball R, Groner B, Friis RR. New mammary epithelial and fibroblastic cell clones in coculture form structures competent to differentiate functionally. J Cell Biol 1989; 108: $1127-1138$
48. Bakiri L, Matsuo K, Wisniewska M, Wagner EF, Yaniv M. Promoter specificity and biological activity of tethered AP-1 dimers. Mol Cell Biol 2002; 22 4952-4964.

49. Bakiri L, Reschke MO, Gefroh HA, Idarraga MH, Polzer K, Zenz R et al. Functions of Fos phosphorylation in bone homeostasis, cytokine response and tumourigenesis. Oncogene 2011; 30: 1506-1517.

50. Maschler S, Wirl G, Spring H, Bredow DV, Sordat I, Beug $H$ et al. Tumor cell invasiveness correlates with changes in integrin expression and localization. Oncogene 2005; 24: 2032-2041.

51. Casalino L, Bakiri L, Talotta F, Weitzman JB, Fusco A, Yaniv M et al. Fra-1 promotes growth and survival in RAS-transformed thyroid cells by controlling cyclin A transcription. EMBO J 2007; 26: 1878-1890.

52. Germain S, Howell M, Esslemont GM, Hill CS. Homeodomain and winged-helix transcription factors recruit activated Smads to distinct promoter elements via a common Smad interaction motif. Genes Dev 2000; 14: 435-451.

Supplementary Information accompanies this paper on Cell Death and Differentiation website (http://www.nature.com/cdd) 